\title{
UNBOUNDED MASS RADIAL SOLUTIONS FOR THE KELLER-SEGEL EQUATION IN THE DISK
}

\author{
DENIS BONHEURE, JEAN-BAPTISTE CASTERAS, AND CARLOS ROMÁN
}

Abstract. We consider the boundary value problem

$$
\left\{\begin{aligned}
-\Delta u+u-\lambda e^{u} & =0, u>0 & & \text { in } B_{1}(0) \\
\partial_{\nu} u & =0 & & \text { on } \partial B_{1}(0)
\end{aligned}\right.
$$

whose solutions correspond to steady states of the Keller-Segel system for chemotaxis. Here $B_{1}(0)$ is the unit disk, $\nu$ the outer normal to $\partial B_{1}(0)$, and $\lambda>0$ is a parameter. We show that, provided $\lambda$ is sufficiently small, there exists a family of radial solutions $u_{\lambda}$ to this system which blow up at the origin and concentrate on $\partial B_{1}(0)$, as $\lambda \rightarrow 0$. These solutions satisfy

$$
\lim _{\lambda \rightarrow 0} \frac{u_{\lambda}(0)}{|\ln \lambda|}=0 \quad \text { and } \quad 0<\lim _{\lambda \rightarrow 0} \frac{1}{|\ln \lambda|} \int_{B_{1}(0)} \lambda e^{u_{\lambda}(x)} d x<\infty,
$$

having in particular unbounded mass, as $\lambda \rightarrow 0$.

Keywords: Keller-Segel equation, singular solution, point concentration, boundary layer, unbounded mass.

MSC: 35B40, 35B45, 35J57, 92C15, 92C40.

\section{INTRODUCTION}

Chemotaxis is the influence of chemical substances in the environment on the movement of mobile species. It is an important mean for cellular communication by chemical substances, which determines how cells arrange themselves, for instance in living tissues. In 1970, Keller and Segel [KS70] proposed a basic model to describe this phenomenon. They considered an advection-diffusion system consisting of two coupled parabolic equations for the concentration of the species and that of the chemical released, respectively represented by strictly positive quantities $v(x, t)$ and $u(x, t)$ defined on a bounded smooth domain $\Omega \subset \mathbb{R}^{n}$. This system has the form

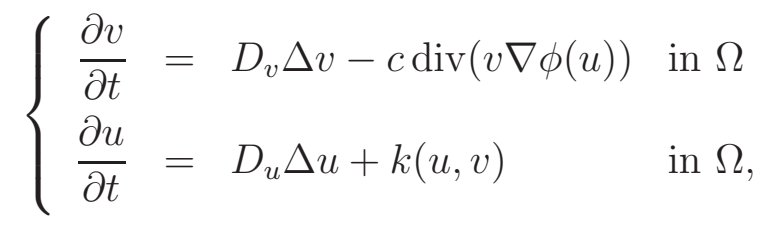

Date: June 1, 2021. 
with no flux through the boundary, that is, letting $\nu$ denote the exterior unit normal vector to $\partial \Omega$,

$$
\nabla v \cdot \nu=\nabla u \cdot \nu=0 \quad \text { on } \partial \Omega .
$$

Here, $D_{v}, D_{u}$, and $c$ are strictly positive constants, the function $\phi$, usually called the sensitive function, is a smooth function such that $\phi^{\prime}(u)>0$ for $u>0$ and $k$ is a smooth function such that $\frac{\partial k}{\partial v} \geq 0$ and $\frac{\partial k}{\partial u} \leq 0$. The typical choice for $k$ that we adopt from now on is $k(u, v)=-u+v$.

An important property of this system is the so-called chemotactic collapse. This term refers to the fact that the whole population of organisms concentrate at a single point in finite or infinite time. When $\phi(u)=u$, it is well-known that the chemotactic collapse depends strongly on the dimension of the space. Finite-time blow-up never occurs if $n=1$, whereas it always occurs if $n \geq 3$. The two-dimensional case is critical: if the initial distribution of organisms exceeds a certain threshold, then the solutions may blow-up in finite time, whereas solutions exist globally in time if the initial mass is below the threshold. We refer the interested reader to the surveys [Hor03, Hor04, BBTW15] and to the references therein for further details about the model and a collection of known results.

Steady states of the Keller-Segel system are of basic importance for the understanding of the global dynamics. They solve the system

$$
\left\{\begin{aligned}
-D_{v} \Delta v+c \operatorname{div}(v \nabla \phi(u)) & =0, \quad v>0 \quad \text { in } \Omega \\
-D_{u} \Delta u-u+v & =0, \quad u>0 \quad \text { in } \Omega
\end{aligned}\right.
$$

with homogeneous Neumann boundary conditions on $\partial \Omega$. This system can be reduced to a scalar equation as, indeed, one easily checks that

$$
\int_{\Omega} v\left|\nabla\left(D_{v} \ln v-c \phi(u)\right)\right|^{2} d x=0
$$

which implies $v=C e^{\frac{c}{D v} \phi(u)}$ for some constant $C>0$. In the most common formulation of the Keller-Segel model, one takes $\phi(u)=u$, which yields the so-called Keller-Segel equation

$$
\left\{\begin{aligned}
-\sigma^{2} \Delta u+u-\lambda e^{u} & =0, u>0 & & \text { in } \Omega \\
\partial_{\nu} u & =0 & & \text { on } \partial \Omega,
\end{aligned}\right.
$$

where the constants $\sigma, \lambda$ depend on $D_{v}, D_{u}, c$ and $C$. It is worth mentioning that in the case $\phi(u)=\ln u$, one gets

$$
\left\{\begin{aligned}
-\tilde{\sigma}^{2} \Delta u+u-u^{p} & =0, u>0 & & \text { in } \Omega \\
\partial_{\nu} u & =0 & & \text { on } \partial \Omega
\end{aligned}\right.
$$

for some constants $\tilde{\sigma}, p>0$, that is, one recovers the celebrated Lin-Ni-Takagi equation [NT86, LN88, LNT88]. Let us observe that in dimension 2 the KellerSegel equation is critical, whereas the Lin-Ni-Takagi problem is subcritical. A good account of known results about this equation is given in the book by Wei and 
Winter [WW14], in the chapter [Ni04], in the recent paper [dPMRW19], and in the references therein.

From now on, we study the Keller-Segel equation (1.1) and we assume without loss of generality that $\sigma=1$. In the one-dimensional case, Schaaf [Sch85] proved the existence of non-trivial solutions. For a general two-dimensional domain, the first existence results were obtained by Wang and Wei [WW02] and independently by Senba and Suzuki [SS00], when the parameter $\lambda$ is small enough. Moreover, Senba and Suzuki [SS00, SS02] studied the asymptotic behavior of finite mass solutions when $\lambda \rightarrow 0$. These are solutions $u_{\lambda}$ to (1.1) such that

$$
\lim _{\lambda \rightarrow 0} \lambda \int_{\Omega} e^{u_{\lambda}}<\infty
$$

They showed that there exist points $\xi_{1}, \ldots, \xi_{k} \in \Omega$ and points $\xi_{k+1}, \ldots, \xi_{k+m} \in \partial \Omega$ such that, in the sense of measures,

$$
-\Delta u_{\lambda}+u_{\lambda}=\lambda e^{u_{\lambda}} \rightarrow \sum_{i=1}^{k} 8 \pi \delta_{\xi_{i}}+\sum_{i=k+1}^{k+m} 4 \pi \delta_{\eta_{i}}
$$

in the sense of measures, and

$$
u_{\lambda}(x) \rightarrow \sum_{i=1}^{k} 8 \pi \mathcal{G}\left(x, \xi_{i}\right)+\sum_{i=k+1}^{m} 4 \pi \mathcal{G}\left(x, \eta_{i}\right), \quad \text { as } \lambda \rightarrow 0,
$$

uniformly on compact subsets of $\bar{\Omega} \backslash\left\{\xi_{1}, \ldots, \xi_{k}, \eta_{k+1}, \ldots, \eta_{m}\right\}$, where, given $y \in \bar{\Omega}$, $\mathcal{G}(x, y)$ denotes the Green function that uniquely solves

$$
\left\{\begin{array}{rll}
-\Delta_{x} \mathcal{G}+\mathcal{G} & =\delta_{y} & \text { in } \Omega \\
\nabla \mathcal{G} \cdot \nu & =0 & \text { on } \partial \Omega
\end{array}\right.
$$

The counterpart of this result was obtained by del Pino and Wei [dPW06]. For any given integers $k$ and $m$, they constructed a family of solutions $u_{\lambda}$ to (1.1) that satisfy (1.2) and (1.3) for a suitable choice of points $\xi_{i} \in \Omega$ for $i=1, \ldots, k$ and $\xi_{i} \in \partial \Omega$ for $i=k+1, \ldots, k+m$. Near each of these points $\xi=\xi_{i}$,

$$
u_{\lambda}(x) \approx V_{\mu_{i}}(|x-\xi|),
$$

where $V_{\mu_{i}}$ is a radially symmetric solution to

$$
-\Delta V-\lambda e^{V}=0 \quad \text { in } \mathbb{R}^{2},
$$

that is, a function of the form

$$
V_{\mu}(|x|)=\ln \frac{8 \mu^{2}}{\left(\lambda \mu^{2}+|x|^{2}\right)^{2}} \quad \text { with } \mu>0 .
$$

The parameter $\mu_{i}=C\left(\xi_{1}, \ldots, \xi_{k+m}, \Omega\right)$ is a constant that depends only on the points $\xi_{i}$ 's and $\Omega$. In particular, it does not depend on $\lambda$. 
It is worth mentioning that these solutions have quantized mass, that is,

$$
\lim _{\lambda \rightarrow 0} \int_{\Omega} \lambda e^{u_{\lambda}}=4 \pi(2 k+m) .
$$

Recently, solutions concentrating on higher dimensional sets with unbounded mass, namely

$$
\lim _{\lambda \rightarrow 0} \int_{\Omega} \lambda e^{u_{\lambda}}=\infty
$$

have been proven to exist. From now on, we denote by $B_{r}$ the ball of radius $r$ centered at zero. When $\Omega=B_{1} \subset \mathbb{R}^{n}$ with $n \geq 2$, Pistoia and Vaira [PV15] constructed a family $u_{\lambda}$ of radial solutions concentrating on the whole boundary of $\Omega$ such that

$$
0<\lim _{\lambda \rightarrow 0} \frac{1}{|\ln \lambda|} \int_{B_{1}} \lambda e^{u_{\lambda}(x)} d x<\infty
$$

More precisely, their solutions satisfy

$$
\lim _{\lambda \rightarrow 0} \frac{1}{|\ln \lambda|} u_{\lambda}=\mathcal{U}
$$

$C^{0}$-uniformly on compact subsets of $B_{1}$, where $\mathcal{U}$ is the unique (radial) solution to

$$
\left\{\begin{array}{rll}
-\Delta \mathcal{U}+\mathcal{U} & =0 & \text { in } B_{1} \\
\mathcal{U} & =1 & \text { on } \partial B_{1} .
\end{array}\right.
$$

whereas near the boundary,

$$
u_{\lambda}(r)+\ln \lambda \approx W_{\varepsilon}(r)=\ln \left(\frac{4}{\varepsilon^{2}} \frac{e^{\sqrt{2} \frac{r-1}{\varepsilon}}}{\left(1+e^{\sqrt{2} \frac{r-1}{\varepsilon}}\right)^{2}}\right),
$$

where $\varepsilon=\varepsilon_{\lambda} \approx \frac{\sqrt{2}}{\mathcal{U}^{\prime}(1)} \frac{1}{|\ln \lambda|}$.

Let us point out that $W_{\varepsilon}$ is a radial solution to the one-dimensional problem

$$
-W^{\prime \prime}=e^{W} \quad \text { in } \mathbb{R}, \quad \text { with } \int_{\mathbb{R}} e^{W}<\infty .
$$

Del Pino, Pistoia, and Vaira [dPPV16], generalized this result to general twodimensional domains. Also, the existence of solutions concentrating on submanifolds of the boundary has also been investigated; see for instance [AP16].

From now on, we suppose that $\Omega=B_{1} \subset \mathbb{R}^{n}$, with $n \geq 2$. In [BCN17b], a bifurcation analysis of radial solutions to (1.1) was performed. Observe that for $\lambda<1 / e$, the equation (1.1) can be rewritten as

$$
\left\{\begin{aligned}
-\Delta u+u & =e^{\mu(u-1)}, u>0 & & \text { in } B_{1} \\
\nabla u \cdot \nu & =0 & & \text { on } \partial B_{1}
\end{aligned}\right.
$$


for $\mu>1$. This equation admits the constant solutions $u \equiv 1$ and $\underline{u}_{\mu}<1$. To describe the bifurcation result, we denote by $\lambda_{i}^{\text {rad }}$ the $i$-th eigenvalue of the operator $-\Delta+\operatorname{Id}$ in $B_{1}$, restricted to the set of radial functions, with homogeneous Neumann boundary conditions.

Theorem ([BCN17b]). For every $i \geq 2,\left(\lambda_{i}^{\mathrm{rad}}, 1\right)$ is a bifurcation point of $(1.6)$. Denoting by $\mathcal{B}_{i}$ the continuum that branches out of $\left(\lambda_{i}^{\mathrm{rad}}, 1\right)$, we have

(i) the branches $\mathcal{B}_{i}$ are unbounded and do not intersect; close to $\left(\lambda_{i}^{\mathrm{rad}}, 1\right), \mathcal{B}_{i}$ is a $C^{1}$-curve;

(ii) if $u_{\mu} \in \mathcal{B}_{i}$ then $u_{\mu}>0$;

(iii) each branch consists of two connected components: the component $\mathcal{B}_{i}^{-}$, along which $u_{\mu}(0)<1$, and the component $\mathcal{B}_{i}^{+}$, along which $u_{\mu}(0)>1$;

(iv) if $u_{\mu} \in \mathcal{B}_{i}$ then $u_{\mu}-1$ has exactly $i-1$ zeros, $u_{\mu}^{\prime}$ has exactly $i-2$ zeros, and each zero of $u_{\mu}^{\prime}$ lies between two zeros of $u_{\mu}-1$;

(v) the functions satisfying $u_{\mu}(0)<1$ are uniformly bounded in the $C^{1}$-norm.

We conjecture that the solutions constructed by Pistoia and Vaira [PV15] correspond to those on $\mathcal{B}_{1}^{-}$, while the solutions constructed by del Pino and Wei [dPW06] (when restricted to the 2-dimensional ball) correspond to the branch $\mathcal{B}_{1}^{+}$. In [BCN17b], the authors constructed solutions concentrating on an arbitrary number of internal spheres by combining variational and perturbative methods. Solutions sharing the same qualitative properties were obtained with a different method in [BCN17a] with very precise asymptotics. We conjecture that those solutions are indeed the same and correspond to the solutions on the branches $\mathcal{B}_{i}^{-}$.

In this paper, we restrict ourselves to the disk, that is, the case $n=2$. Our main result is the construction of solutions to (1.1) that concentrate at the origin and on the boundary of $B_{1}$, as $\lambda \rightarrow 0$. We conjecture that they correspond to the solutions to (1.6) on the branch $\mathcal{B}_{2}^{+}$. We emphasize that only a few results concerning existence of solutions concentrating simultaneously on points and layers are available in the literature, see for instance [SW13, WW08].

Theorem 1.1. There exist $\lambda_{0}>0$ and a family of radial solutions $\left\{u_{\lambda} \mid \lambda \in\left(0, \lambda_{0}\right)\right\}$ to (1.1) such that

$$
\lim _{\lambda \rightarrow 0} \frac{u_{\lambda}(0)}{|\ln \lambda|}=\infty \quad \text { and } \quad 0<\lim _{\lambda \rightarrow 0} \frac{1}{|\ln \lambda|} \int_{B_{1}} \lambda e^{u_{\lambda}(x)} d x<\infty
$$

Moreover, letting $\varepsilon_{\lambda} \rightarrow 0$ as $\lambda \rightarrow 0$ be the parameter defined by

$$
\ln \frac{4}{\varepsilon_{\lambda}^{2}}-\ln \lambda=\frac{a_{1, \varepsilon_{\lambda}}}{\varepsilon_{\lambda}}+a_{2, \varepsilon_{\lambda}}+a_{3, \varepsilon_{\lambda}} \varepsilon_{\lambda}
$$


for suitable constants $a_{1, \varepsilon_{\lambda}}, a_{2, \varepsilon_{\lambda}}$, and $a_{3, \varepsilon_{\lambda}}$ depending on $\varepsilon_{\lambda}$ (see (3.7)), and letting $G_{\varepsilon_{\lambda}}$ be the unique radial solution for $\varepsilon=\varepsilon_{\lambda}$ to

$$
\left\{\begin{array}{rlrl}
-\Delta G_{\varepsilon}+G_{\varepsilon} & =0 & & \text { in } B_{1} \\
\lim _{r \rightarrow 0^{+}} \frac{G_{\varepsilon}(r)}{|\ln r|} & =4 / \mathcal{A}_{\varepsilon} & \\
G_{\varepsilon} & =1 & \text { on } \partial B_{1}
\end{array}\right.
$$

where $\mathcal{A}_{\varepsilon}=\frac{A_{1, \varepsilon}}{\varepsilon}+A_{2, \varepsilon}+A_{3, \varepsilon} \varepsilon$ for suitable constants $A_{1, \varepsilon}, A_{2, \varepsilon}$, and $A_{3, \varepsilon}$ depending on $\varepsilon$ (see (3.6)), we have that, uniformly on compact subsets of $B_{1} \backslash\{0\}$,

$$
\lim _{\lambda \rightarrow 0}\left(u_{\lambda}(x)-\mathcal{A}_{\varepsilon_{\lambda}} G_{\varepsilon_{\lambda}}(x)\right)=0,
$$

and, in the sense of measures,

$$
\lambda e^{u_{\lambda}} \rightarrow 8 \pi \delta_{0} \quad \text { in } B_{1 / 2}, \quad \varepsilon_{\lambda} \lambda e^{u_{\lambda}} \rightarrow \sqrt{2} \delta_{\partial B_{1}} \quad \text { in } \bar{B}_{1} \backslash B_{1 / 2} .
$$

Theorem 1.1 will be proven using a fixed point argument. More precisely, we will look for a solution to (1.1) of the form $u_{\lambda}=U_{\lambda}+\phi_{\lambda}$, where $U_{\lambda}$ is a first "good" approximation of the solution and $\phi_{\lambda}$ is a small perturbation. Roughly speaking, $U_{\lambda}$ is constructed by gluing the Green's function $\mathcal{A}_{\varepsilon_{\lambda}} G_{\varepsilon_{\lambda}}$ with $V_{\mu}$ (recall (1.4)) near the origin and with $W_{\varepsilon}$ (recall (1.5)) near the boundary, for well chosen parameters $\mu$ and $\varepsilon$. To obtain a "good" matching between these functions, we are forced to choose $\varepsilon_{\lambda}$ satisfying (1.7) and

$$
\mu^{2}=\mu_{\lambda}^{2}=\frac{e^{H_{\varepsilon_{\lambda}}(0)}}{8}
$$

where $H_{\varepsilon_{\lambda}}$ denotes the regular part of $\mathcal{A}_{\varepsilon_{\lambda}} G_{\varepsilon_{\lambda}}$, that is $H_{\varepsilon_{\lambda}}(r)=\mathcal{A}_{\varepsilon_{\lambda}} G_{\varepsilon_{\lambda}}(r)+4 \ln r$. We explicitly compute $H_{\varepsilon_{\lambda}}(0)$ (see (3.11)), which leads to

$$
8 \mu_{\lambda}^{2} \approx e^{\frac{A_{1}}{C \varepsilon_{\lambda}}} \rightarrow \infty \quad \text { as } \lambda \rightarrow 0
$$

where $A_{1}=\lim _{\varepsilon \rightarrow 0} A_{1, \varepsilon}>0$ and $C>1$ is a constant. Let us stress that this is very different to the previously described situation of finite mass blow-up, and poses technical issues. In fact, what allows our argument to work is the crucial fact that

$$
\lim _{\lambda \rightarrow 0} \lambda \mu_{\lambda}^{2}=\lim _{\lambda \rightarrow 0} \frac{1}{\varepsilon_{\lambda}^{2}} \exp \left(\frac{A_{1}}{\varepsilon_{\lambda}}\left(\frac{1}{C}-1\right)\right)=0 .
$$

The rest of the paper is organized as follows. In Section 2, we provide the existence of the Green's function solution to (1.8), which is used to build the first approximation of the solution in Section 3. We then estimate the error introduced by our approximation in Section 4. In Section 5, we prove the solvability of the linearized equation around our approximate solution, which allows us to use a fixed point argument to prove Theorem 1.1 in Section 6. 


\section{ACKNOWLEDGMENTS}

D. Bonheure is partially supported by the project ERC Advanced Grant $2013 \mathrm{n}$. 339958: "Complex Patterns for Strongly Interacting Dynamical Systems - COMPAT" and by ARC AUWB-2012-12/17-ULB1- IAPAS.

J-B. Casteras is supported by MIS F.4508.14 (FNRS), PDR T.1110.14F (FNRS) and INRIA Team MEPHYSTO.

This work was initiated when C. Román was a Ph.D. student at the JacquesLouis Lions Laboratory of the Pierre and Marie Curie University, supported by a public grant overseen by the French National Research Agency (ANR) as part of the "Investissements d'Avenir" program (reference: ANR-10-LABX-0098, LabEx SMP). He is currently supported by the Chilean National Agency for Research and Development (ANID) through FONDECYT Iniciación grant 11190130. He wishes to thank the support and kind hospitality of the Université libre de Bruxelles, where part of this work was done.

\section{GREen's FUnCTION}

This section is devoted to prove the existence of $G_{\varepsilon}$ defined in (1.8). First, let us recall the following lemma from [BCN17b].

Lemma 2.1. There exist two positive linearly independent solutions $\zeta \in C^{2}((0,1])$ and $\xi \in C^{2}([0,1])$ of the modified zero-order Bessel differential equation

$$
-u^{\prime \prime}-\frac{1}{r} u^{\prime}+u=0 \quad \text { in }(0,1)
$$

satisfying

$$
\xi^{\prime}(0)=\zeta^{\prime}(1)=0 \quad \text { and } \quad r\left(\xi^{\prime}(r) \zeta(r)-\xi(r) \zeta^{\prime}(r)\right)=1 \text { for any } r \in(0,1] .
$$

We have that $\xi$ is bounded and strictly increasing in $[0,1], \zeta$ is strictly decreasing in $(0,1]$

$$
\xi(0)=1, \quad \lim _{r \rightarrow 0^{+}} \frac{\zeta(r)}{|\ln r|}=1, \quad \text { and } \quad \lim _{r \rightarrow 0^{+}}\left(-r \zeta^{\prime}(r)\right)=1 .
$$

Moreover, as $r$ goes to 0, we have (see [AS64])

$$
\begin{gathered}
\zeta(r)=\left(|\ln r|+c_{1}\right)+\frac{r^{2}}{4}\left(|\ln r|+c_{2}\right)+O\left(r^{4}|\ln r|\right), \\
\zeta^{\prime}(r)=-\frac{1}{r}+\frac{r\left(|\ln r|+c_{3}\right)}{2}+O\left(r^{3}|\ln r|\right),
\end{gathered}
$$

and

$$
\xi(r)=1+\frac{r^{2}}{4}+O\left(r^{4}\right), \quad \xi^{\prime}(r)=\frac{r}{2}+O\left(r^{3}\right),
$$

where $c_{1}, c_{2}, c_{3}$ are positive constants. 
Using this result, we are able to construct a radial Green's function on the unit ball $B_{1}$ blowing up at 0 and equal to 1 on $\partial B_{1}$.

Lemma 2.2. For any $\varepsilon>0$ small enough, there exists a function $G_{\varepsilon}$ satisfying

$$
\left\{\begin{aligned}
-G_{\varepsilon}^{\prime \prime}-\frac{1}{r} G_{\varepsilon}^{\prime}+G_{\varepsilon} & =0 \quad \text { in }(0,1) \\
\lim _{r \rightarrow 0^{+}} \frac{G_{\varepsilon}(r)}{|\ln r|} & =4 / \mathcal{A}_{\varepsilon} \\
G_{\varepsilon}(1) & =1
\end{aligned}\right.
$$

where $\mathcal{A}_{\varepsilon}=\frac{A_{1, \varepsilon}}{\varepsilon}+A_{2, \varepsilon}+A_{3, \varepsilon} \varepsilon$, with

$$
A_{1, \varepsilon}=\frac{\sqrt{2}}{G_{\varepsilon}^{\prime}(1)}, A_{2, \varepsilon}=\frac{1}{G_{\varepsilon}^{\prime}(1)}\left(\frac{\ln 4}{G_{\varepsilon}^{\prime}(1)}-2\right), A_{3, \varepsilon}=\frac{c}{G_{\varepsilon}^{\prime}(1)}
$$

for $c \in \mathbb{R}$.

Moreover, there exists $\tilde{r} \in(0,1)$ with $\tilde{r} \approx \sqrt{\varepsilon}$, that is, there exist two constants $c_{1}, c_{2}>0$ such that $c_{1} \sqrt{\varepsilon} \leq \tilde{r} \leq c_{2} \sqrt{\varepsilon}$, such that $G_{\varepsilon}^{\prime}(\tilde{r})=0$, and there holds $\lim _{\varepsilon \rightarrow 0} G_{\varepsilon}^{\prime}(1)=\frac{\xi^{\prime}(1)}{\xi(1)}$. We also have, as $r$ goes to zero,

$$
G_{\varepsilon}(r)-\frac{4}{\mathcal{A}_{\varepsilon}}|\ln r|=\frac{1}{\xi(1)}+O_{r}\left(r^{2}\right)+\frac{O_{r}\left(r^{2}|\ln r|\right)}{\mathcal{A}_{\varepsilon}}+O_{\varepsilon}(\varepsilon)
$$

where $C$ is a constant independent of $\varepsilon$, and

$$
G_{\varepsilon}^{\prime}(r)+\frac{4}{\mathcal{A}_{\varepsilon} r}=O(r)+\frac{O(r|\ln r|)}{\mathcal{A}_{\varepsilon}} .
$$

Proof. Using the properties of the functions $\xi$ and $\zeta$ (defined in Lemma 2.1), it is immediate to see that, for any $b \in(0,1)$,

$$
u_{b}(r)=\frac{\xi^{\prime}(b) \zeta(r)-\xi(r) \zeta^{\prime}(b)}{\xi^{\prime}(b) \zeta(1)-\xi(1) \zeta^{\prime}(b)}
$$

is a solution to $(2.4)$ such that

$$
u_{b}(1)=1 \quad \text { and } \quad \lim _{r \rightarrow 0^{+}} \frac{u_{b}(r)}{|\ln r|}=\frac{\xi^{\prime}(b)}{\xi^{\prime}(b) \zeta(1)-\xi(1) \zeta^{\prime}(b)} .
$$

Moreover, for $b$ small enough, we have

$$
\begin{aligned}
\xi^{\prime}(b) & =\frac{1}{2} b+o(b), \\
\xi^{\prime}(b) \zeta(1)-\xi(1) \zeta^{\prime}(b) & =\xi(1) b^{-1}+O(b) .
\end{aligned}
$$

Therefore, for $b$ small enough, we have

$$
\lim _{r \rightarrow 0^{+}} \frac{u_{b}(r)}{|\ln r|}=\frac{1}{2 \xi(1)} b^{2}+o\left(b^{2}\right) .
$$


On the other hand, it is easy to check that

$$
u_{b}^{\prime}(1)=\frac{\xi^{\prime}(1)}{\xi(1)}+o_{b}(1)
$$

where $o_{b}(1) \rightarrow 0$ as $b \rightarrow 0$. Therefore, we can choose $b \approx \sqrt{\varepsilon}$ such that $\lim _{r \rightarrow 0^{+}} \frac{u_{b}(r)}{|\ln r|}=$ $4 / \mathcal{A}_{\varepsilon}$, which proves the existence of the function $G_{\varepsilon}$.

From $(2.7)$, we immediately see that $\lim _{\varepsilon \rightarrow 0} G_{\varepsilon}^{\prime}(1)=\frac{\xi^{\prime}(1)}{\xi(1)}$. The fact that $u_{b}^{\prime}(b)=0$ implies the existence of $\tilde{r} \approx \sqrt{\varepsilon}$ such that $G_{\varepsilon}^{\prime}(\tilde{r})=0$. Finally, (2.5) and (2.6) follow from (2.1), (2.2), and (2.3).

\section{The Approximate solution}

We look for a radial solution to (1.1) concentrating at 0 and on $\partial B_{1}$. To do so, we take an ansatz of solution of the form

$$
U= \begin{cases}u_{0} & \text { in }[0, \delta) \\ u_{1} & \text { in }[\delta, 2 \delta) \\ u_{2} & \text { in }\left[2 \delta, 1-2 \delta_{1}\right) \\ u_{3} & \text { in }\left[1-2 \delta_{1}, 1-\delta_{1}\right) \\ u_{4} & \text { in }\left[1-\delta_{1}, 1\right],\end{cases}
$$

where $\delta$ and $\delta_{1}$ are suitable constants depending on $\lambda$. Let us first describe our ansatz in words. Near the origin, we want $U=u_{0}$ to behave approximately like $V_{\mu}$, the two dimensional standard bubble given by

$$
V_{\mu}(r)=\ln \frac{8 \mu^{2}}{\left(\lambda \mu^{2}+r^{2}\right)^{2}},
$$

for some constant $\mu=\mu_{\lambda}>0$ to be specified later. Let us recall that these functions correspond to all solutions of the problem

$$
-\Delta V=\lambda e^{V} \quad \text { in } \mathbb{R}^{2}, \quad \text { with } \lambda \int_{\mathbb{R}^{2}} e^{V} d x<\infty .
$$

Near the boundary $\partial B_{1}$ of the disk, we want that $U=u_{4}$ behaves like $W_{\varepsilon}-\ln \lambda$ where $W_{\varepsilon}$ is the one dimensional standard bubble solving $-W^{\prime \prime}=e^{W}$ in $\mathbb{R}$, which is given by

$$
W_{\varepsilon}(r)=\ln \left(\frac{4}{\varepsilon^{2}} \frac{e^{-\frac{\sqrt{2}(r-1)}{\varepsilon}}}{\left(1+e^{-\frac{\sqrt{2}(r-1)}{\varepsilon}}\right)^{2}}\right),
$$

for some constant $\varepsilon=\varepsilon_{\lambda}>0$ to be determined later. In order to "glue" these singular solutions, far from the origin and $\partial B_{1}$ we choose $U=\mathcal{A}_{\varepsilon_{\lambda}} G_{\varepsilon_{\lambda}}$, where $G_{\varepsilon_{\lambda}}$ is the singular at the origin Green's function given in Lemma 2.2 (with $\varepsilon=\varepsilon_{\lambda}$ and a 
suitable constant $c$ ). Finally, we choose $u_{1}$ and $u_{3}$ to be linear interpolations between $u_{i-1}$ and $u_{i+1}$, for $i=1,3$, namely

$$
u_{i}(r)=\chi_{i}(r) u_{i-1}(r)+\left(1-\chi_{i}(r)\right) u_{i+1}(r),
$$

where $\chi_{i} \in C^{2}((0,1))$ are cut-off functions such that

$\chi_{1}(r) \equiv 1$ in $(0, \delta), \chi_{1}(r) \equiv 0$ in $(2 \delta, 1),\left|\chi_{1}(r)\right| \leq 1,\left|\chi_{1}^{\prime}(r)\right| \leq c \delta^{-1},\left|\chi_{1}^{\prime \prime}(r)\right| \leq c \delta^{-2}$,

and

$\chi_{3} \equiv 1$ in $\left(0,1-2 \delta_{1}\right), \chi_{3} \equiv 0$ in $\left(1-\delta_{1}, 1\right),\left|\chi_{3}(r)\right| \leq 1,\left|\chi_{3}^{\prime}(r)\right| \leq c \delta_{1}^{-1},\left|\chi_{3}^{\prime \prime}(r)\right| \leq c \delta_{1}^{-2}$.

We now describe our ansatz in detail.

3.1. Construction of $u_{4}$. First, let us set $\varepsilon=\varepsilon_{\lambda} \rightarrow 0$ as $\lambda \rightarrow 0$, via the relation

$$
\ln \frac{4}{\varepsilon^{2}}-\ln \lambda=\frac{a_{1, \varepsilon}}{\varepsilon}+a_{2, \varepsilon}+a_{3, \varepsilon} \varepsilon
$$

for some constants $a_{i, \varepsilon}, i=1,2,3$ to be determined later (see (3.7)), and let

$$
\delta_{1}=\varepsilon^{\eta}, \text { for some } \eta \in\left(\frac{2}{3}, 1\right) .
$$

We define $u_{4}$ in the same way as the function " $u_{1}$ " of [PV15] (or [BCN17a]) with $r_{0}=1$. The construction of this function is quite lengthy .We only briefly recall it here, and refer to the above two papers for more details. We define

$$
u_{4}=\underbrace{W_{\varepsilon}-\ln \lambda+\alpha_{\varepsilon}}_{1^{\text {st }} \text { order approx. }}+\underbrace{v_{\varepsilon}+\beta_{\varepsilon}}_{2^{\text {nd }} \text { order }}+\underbrace{z_{\varepsilon}}_{3^{\text {rd }} \text { order }},
$$

where $\alpha_{\varepsilon}(r), v_{\varepsilon}(r), \beta_{\varepsilon}(r)$, and $z_{\varepsilon}(r)$, which we briefly describe below, are functions introduced in order to produce a good enough match between $u_{4}$ and $u_{2}=\mathcal{A}_{\varepsilon} G_{\varepsilon}$; see Lemma 3.1.

The function $\alpha_{\varepsilon}$ satisfies

$$
\left\{\begin{aligned}
-\left(\alpha_{\varepsilon}\right)^{\prime \prime}-\frac{1}{r}\left(\alpha_{\varepsilon}\right)^{\prime} & =\frac{1}{r}\left(W_{\varepsilon}\right)^{\prime}-W_{\varepsilon}+\ln \lambda \text { in }(0,1) \\
\alpha_{\varepsilon}(1) & =0 \\
\left(\alpha_{\varepsilon}\right)^{\prime}(1) & =0
\end{aligned}\right.
$$

and the following estimate holds, for $s \leq 0$,

$$
\alpha_{\varepsilon}(\varepsilon s+1)=\varepsilon\left(\alpha_{\varepsilon}\right)_{1}(s)+\varepsilon^{2}\left(\alpha_{\varepsilon}\right)_{2}(s)+O\left(\varepsilon^{3} s^{4}\right),
$$

where, letting $W$ be defined via

$$
W\left(\frac{r-1}{\varepsilon}\right)+\ln \frac{4}{\varepsilon^{2}}-\ln 4=W_{\varepsilon}(r)
$$

we have

$$
\left(\alpha_{\varepsilon}\right)_{1}(s)=-\int_{0}^{s} W(\sigma) d \sigma+\frac{a_{1, \lambda}}{2} s^{2}
$$


and

$$
\left(\alpha_{\varepsilon}\right)_{2}(s)=\int_{0}^{s} \int_{0}^{\sigma}(W(\rho)-\ln 4) d \rho d \sigma+\int_{0}^{s} \sigma W(\sigma) d \sigma-\frac{1}{6} a_{1, \lambda} s^{3}+\frac{a_{2, \lambda}}{2} s^{2} .
$$

The function $v_{\varepsilon}$ solves

$$
\left\{\begin{aligned}
-\left(v_{\varepsilon}\right)^{\prime \prime}-e^{W_{\varepsilon}} v_{\varepsilon} & =\varepsilon e^{W_{\varepsilon}}\left(\alpha_{\varepsilon}\right)_{1}\left(\frac{r-1}{\varepsilon}\right) \text { in } \mathbb{R} \\
v_{\varepsilon}(1) & =0 \\
\left(v_{\varepsilon}\right)^{\prime}(1) & =0
\end{aligned}\right.
$$

where $\left(\alpha_{\varepsilon}\right)_{1}$ is defined in (3.5). Moreover, we have

$$
v_{\varepsilon}(r)=\nu_{1}(r-1)+\nu_{2} \varepsilon+O\left(\varepsilon e^{-\frac{|r-1|}{\varepsilon}}\right),
$$

where

$$
\nu_{2} \in \mathbb{R} \quad \text { and } \quad \nu_{1}=-2(1-\ln 2)+a_{1} \sqrt{2} \ln 2 .
$$

The function $\beta_{\varepsilon}$ is the solution of

$$
\left\{\begin{aligned}
-\left(\beta_{\varepsilon}\right)^{\prime \prime}-\frac{1}{r}\left(\beta_{\varepsilon}\right)^{\prime} & =\frac{1}{r}\left(v_{\varepsilon}\right)^{\prime} \quad \text { in }(0,1), \\
\beta_{\varepsilon}(1) & =0 \\
\left(\beta_{\varepsilon}\right)^{\prime}(1) & =0
\end{aligned}\right.
$$

and the following estimate holds, for $s \leq 0$,

$$
\beta_{\varepsilon}(\varepsilon s+1)=\varepsilon^{2}\left(\beta_{\varepsilon}\right)_{1}(s)+O\left(\varepsilon^{3} s^{3}\right),
$$

where, letting $v$ be defined via

$$
v_{\varepsilon}(r)=\varepsilon v\left(\frac{r-1}{\varepsilon}\right),
$$

we have

$$
\left(\beta_{\varepsilon}\right)_{1}(s)=-\int_{0}^{s} \int_{0}^{\sigma} v^{\prime}(\rho) d \rho d \sigma
$$

Finally, the function $z_{\varepsilon}$ satisfies

$$
\left\{\begin{aligned}
-\left(z_{\varepsilon}\right)^{\prime \prime}-e^{W_{\varepsilon}} z_{\varepsilon} & =\varepsilon^{2} e^{W_{\varepsilon}}\left[\left(\alpha_{\varepsilon}\right)_{2}\left(\frac{r-1}{\varepsilon}\right)+\left(\beta_{\varepsilon}\right)_{1}\left(\frac{r-1}{\varepsilon}\right)\right. \\
& \\
& \left.+\frac{1}{2}\left(\left(\alpha_{\varepsilon}\right)_{1}\left(\frac{r-1}{\varepsilon}\right)+v\left(\frac{r-1}{\varepsilon}\right)\right)^{2}\right] \text { in }(0,1) \\
z_{\varepsilon}(1) & =0 \\
\left(z_{\varepsilon}\right)^{\prime}(1) & =0
\end{aligned}\right.
$$

and there holds

$$
z_{\varepsilon}(r)=\varepsilon \zeta_{1}(r-1)+\zeta_{2} \varepsilon^{2}+O\left(\varepsilon^{2} e^{-\frac{|r-1|}{\varepsilon}}\right)
$$

for some $\zeta_{j} \in \mathbb{R}, j=1,2$. 
3.2. Construction of $u_{2}$. Thanks to Lemma 2.2 (with $c=\zeta_{1}$ ), we know that, for any $\varepsilon$ small enough, there exists a function $G_{\varepsilon}$ satisfying

$$
\left\{\begin{aligned}
-G_{\varepsilon}^{\prime \prime}-\frac{1}{r} G_{\varepsilon}^{\prime}+G_{\varepsilon} & =0 \quad \text { in }(0,1) \\
\lim _{r \rightarrow 0^{+}} \frac{G_{\varepsilon}(r)}{|\ln r|} & =4 / \mathcal{A}_{\varepsilon} \\
G_{\varepsilon}(1) & =1 .
\end{aligned}\right.
$$

where $\mathcal{A}_{\varepsilon}=\frac{A_{1, \varepsilon}}{\varepsilon}+A_{2, \varepsilon}+A_{3, \varepsilon} \varepsilon$ and

$$
A_{1, \varepsilon}=\frac{\sqrt{2}}{G_{\varepsilon}^{\prime}(1)}, \quad A_{2, \varepsilon}=\frac{1}{G_{\varepsilon}^{\prime}(1)}\left(\frac{\ln 4}{G_{\varepsilon}^{\prime}(1)}-2\right), \quad A_{3, \varepsilon}=\frac{\zeta_{1}}{G_{\varepsilon}^{\prime}(1)} .
$$

Letting

$$
a_{1, \varepsilon}=A_{1, \varepsilon}, \quad a_{2, \varepsilon}=A_{2, \varepsilon}, \quad a_{3, \varepsilon}=A_{3, \varepsilon}-\nu_{2},
$$

and recalling (3.3), we define

$$
u_{2}(r)=\mathcal{A}_{\varepsilon_{\lambda}} G_{\varepsilon_{\lambda}}(r) .
$$

Thanks to our definition of $u_{2}$ and $u_{4}$, one can show, arguing as in [BCN17a, Lemma 3.3], the following estimates.

Lemma 3.1. For any $\delta_{1}<|r-1|<2 \delta_{1}$, we have

$$
u_{4}(r)-u_{2}(r)=O\left(\varepsilon_{\lambda}^{2}+\varepsilon_{\lambda}|r-1|^{2}+|r-1|^{3}+\frac{|r-1|^{4}}{\varepsilon_{\lambda}}+\exp \left(-\frac{|r-1|}{\varepsilon_{\lambda}}\right)\right)
$$

and

$$
u_{4}^{\prime}(r)-u_{2}^{\prime}(r)=O\left(\varepsilon_{\lambda}|r-1|+|r-1|^{2}+\frac{|r-1|^{3}}{\varepsilon_{\lambda}}+\frac{1}{\varepsilon_{\lambda}} \exp \left(-\frac{|r-1|}{\varepsilon_{\lambda}}\right)\right) .
$$

In order to define $u_{0}$ and estimate $u_{0}-u_{2}$, it is important to introduce the regular part $H_{\varepsilon_{\lambda}}$ of $u_{2}$, namely

$$
H_{\varepsilon_{\lambda}}(r)=u_{2}(r)+4 \ln r
$$

We let $\mu_{\lambda}>0$ be defined via the relation

$$
8 \mu_{\lambda}^{2}=e^{H_{\varepsilon_{\lambda}}(0)} .
$$

Thanks to $(2.5),(2.6)$, and $\lim _{\lambda \rightarrow 0} G_{\varepsilon_{\lambda}}^{\prime}(1)=\frac{\xi^{\prime}(1)}{\xi(1)}$, we have, for a constant $c$ independent of $\varepsilon_{\lambda}$,

$$
H_{\varepsilon_{\lambda}}(0)=\frac{\sqrt{2}}{\xi^{\prime}(1) \varepsilon_{\lambda}}+c+O_{\varepsilon_{\lambda}}\left(\varepsilon_{\lambda}\right) \text { and } \lim _{r \rightarrow 0^{+}} H_{\varepsilon_{\lambda}}^{\prime}(r)=0 .
$$


Moreover, as $r \rightarrow 0$, we have

$$
\left|H_{\varepsilon_{\lambda}}(r)-H_{\varepsilon_{\lambda}}(0)\right| \leq C\left(\frac{r^{2}}{\varepsilon_{\lambda}}+r^{2}|\ln r|\right)
$$

where $C>0$ is a constant independent of $\varepsilon_{\lambda}$.

In particular, recalling (3.3), we have the crucial estimate

$$
\lambda \mu_{\lambda}^{2} \approx \frac{C}{\varepsilon_{\lambda}^{2}} \exp \left(\frac{A_{1}}{\varepsilon_{\lambda}}\left(\frac{1}{\xi(1)}-1\right)\right) \rightarrow 0 \quad \text { as } \lambda \rightarrow 0 \text { (and thus } \varepsilon_{\lambda} \rightarrow 0 \text { ) }
$$

where $A_{1}=\lim _{\lambda \rightarrow 0} A_{1, \varepsilon_{\lambda}}>0, C>0$ is a constant independent of $\varepsilon_{\lambda}$, and $\xi$ is the function defined in Lemma 2.1, which satisfies $\xi(1)>1$ due to the fact that it is an strictly increasing function with $\xi(0)=1$.

3.3. Construction of $u_{0}$. Recall, by Lemma 2.2, that there exists

$$
\tilde{r} \approx \sqrt{\varepsilon}_{\lambda}
$$

such that $u_{2}^{\prime}(\tilde{r})=0$. We define

$$
u_{0}=V_{\mu_{\lambda}}+H_{0, \mu_{\lambda}},
$$

where $V_{\mu_{\lambda}}$ is the function defined in (3.1) and $H_{0, \mu_{\lambda}}$ is the solution, for $\mu=\mu_{\lambda}$, to

$$
\left\{\begin{aligned}
-\Delta H_{0, \mu}+H_{0, \mu} & =-V_{\mu} \quad \text { in }(0, \tilde{r}) \\
H_{0, \mu}^{\prime}(\tilde{r}) & =-V_{\mu}^{\prime}(\tilde{r}) .
\end{aligned}\right.
$$

We introduced the function $H_{0, \mu_{\lambda}}$ in order to get a better matching between $u_{0}$ and $u_{2}$. We choose $\delta$ such that

$$
2 \delta<\tilde{r} \text { and } \delta \approx \sqrt{\varepsilon_{\lambda}} \text {. }
$$

Arguing in a similar way to the proof of [dPW06, Lemma 2.1], we obtain the following estimates.

Lemma 3.2. For any $\alpha \in\left(0, \frac{1}{2}\right)$, we have, for $r \in(0, \tilde{r})$,

$$
H_{0, \mu_{\lambda}}(r)=H_{\varepsilon_{\lambda}}(r)-\ln \left(8 \mu_{\lambda}^{2}\right)+O\left(\left(\mu_{\lambda}^{2} \lambda\right)^{\alpha}\right)
$$

$C^{0, \gamma}\left(B_{\tilde{r}}\right)$-uniformly, for $\gamma \in[0,1)$, where $H_{\varepsilon_{\lambda}}(r)$ is defined in (3.9). Moreover, (3.18) holds uniformly in $C^{1}\left(B_{2 \delta} \backslash B_{\delta}\right)$. Finally, for $r \in(0, \tilde{r})$,

$$
\left|H_{0, \mu_{\lambda}}(r)\right| \leq C\left(\frac{r^{2}}{\varepsilon_{\lambda}}+r^{2}|\ln r|+\left(\lambda \mu_{\lambda}^{2}\right)^{\alpha}\right)
$$

where $C>0$ is a constant independent of $\varepsilon_{\lambda}$.

Proof. Let us consider the function $z(r)=H_{0, \mu_{\lambda}}(r)-H_{\varepsilon_{\lambda}}(r)+\ln \left(8 \mu_{\lambda}^{2}\right)$, which satisfies

$$
\left\{\begin{aligned}
-\Delta z+z & =-\ln \frac{1}{\left(\lambda \mu_{\lambda}^{2}+r^{2}\right)^{2}}+\ln \frac{1}{r^{4}} \text { in }(0, \tilde{r}) \\
z^{\prime}(\tilde{r}) & =\frac{4 \tilde{r}}{\lambda \mu_{\lambda}^{2}+\tilde{r}^{2}}-\frac{4}{\tilde{r}} .
\end{aligned}\right.
$$


By recalling $(3.3)$ and that $\tilde{r} \approx \sqrt{\varepsilon}$, we deduce that

$$
z^{\prime}(\tilde{r})=-\frac{4 \lambda \mu_{\lambda}^{2}}{\tilde{r}\left(\lambda \mu_{\lambda}^{2}+\tilde{r}^{2}\right)}=O\left(\left(\lambda \mu_{\lambda}^{2}\right)^{\alpha}\right)
$$

for any $\alpha \in(0,1)$. We set $f=-\ln \frac{1}{\left(\lambda \mu_{\lambda}^{2}+r^{2}\right)^{2}}+\ln \frac{1}{r^{4}}$ and let $p>2$. We have

$$
\int_{B_{\tilde{r}}}|f|^{p} d x=\int_{B_{\tilde{r}} \backslash B_{\sqrt{\lambda} \mu_{\lambda}}}|f|^{p} d x+\int_{B_{\sqrt{\lambda} \mu_{\lambda}}}|f|^{p} d x .
$$

It is easy to see that

$$
\int_{B_{\sqrt{\lambda} \mu_{\lambda}}}|f|^{p} d x \leq C \lambda \mu_{\lambda}^{2}\left|\ln \left(\lambda \mu_{\lambda}^{2}\right)\right|^{p}
$$

and, using the fact that $|f(r)| \leq \frac{C \sqrt{\lambda} \mu_{\lambda}}{r}$, one gets

$$
\int_{B_{\tilde{r} \backslash B \sqrt{\lambda} \mu_{\lambda}}}|f|^{p} d x \leq C \lambda^{p / 2} \mu_{\lambda}^{p}\left(\sqrt{\lambda} \mu_{\lambda}\right)^{2-p} \leq C \lambda \mu_{\lambda}^{2} .
$$

Using elliptic regularity theory (see Lemma A.1), we deduce that

$$
\|z\|_{C^{0, \gamma}\left(B_{\tilde{r}}\right)} \leq C\left(\lambda \mu_{\lambda}^{2}\right)^{\alpha}
$$

for any $\gamma \in(0,1)$ and $\alpha \in\left(0, \frac{1}{2}\right)$.

On the other hand, for any $q \geq 2$, since $\delta \approx \sqrt{\varepsilon_{\lambda}}$, we have

$$
\int_{B_{2 \delta} \backslash B_{\delta}}|f|^{q} d x \leq C\left(\lambda \mu_{\lambda}^{2}\right)^{q / 2} \delta^{2-q} \leq C\left(\lambda \mu_{\lambda}^{2}\right)^{q / 2} \varepsilon_{\lambda}^{\frac{1}{2}(2-q)} \leq C\left(\lambda \mu_{\lambda}^{2}\right)^{\alpha q},
$$

for any $\alpha \in\left(0, \frac{1}{2}\right)$. We deduce that

$$
\|z\|_{C^{1}\left(B_{2 \delta} \backslash B_{\delta}\right)} \leq C\left(\lambda \mu_{\lambda}^{2}\right)^{\alpha} .
$$

Finally, (3.19) is a direct consequence of (3.18) and (3.12).

Thanks to the previous lemma, we are able to show that $u_{0}$ and $u_{2}$ are very close in $C^{1}$-norm sense in the interval $[\delta, 2 \delta]$.

Lemma 3.3. For $\delta \leq r \leq 2 \delta$, we have

$$
\left|u_{0}(r)-u_{2}(r)\right|=O\left(\left(\lambda \mu_{\lambda}^{2}\right)^{\alpha}\right) \quad \text { and } \quad\left|u_{0}^{\prime}(r)-u_{2}^{\prime}(r)\right|=O\left(\left(\lambda \mu_{\lambda}^{2}\right)^{\alpha}\right)
$$

for any $\alpha \in\left(0, \frac{1}{2}\right)$.

Proof. The proof is a direct consequence of Lemma 3.2. Indeed, by definition, for $r \in[\delta, 2 \delta]$, we have

$$
u_{0}(r)=V_{\mu_{\lambda}}(r)+H_{0, \mu_{\lambda}}(r)=\ln \frac{8 \mu_{\lambda}^{2}}{\left(\lambda \mu_{\lambda}^{2}+r^{2}\right)^{2}}+H_{\varepsilon_{\lambda}}(r)-\ln \left(8 \mu_{\lambda}^{2}\right)+O\left(\left(\lambda \mu_{\lambda}^{2}\right)^{\alpha}\right)
$$


and

$$
u_{2}(r)=-4 \ln r+H_{\varepsilon_{\lambda}}(r) .
$$

It follows that

$$
\begin{aligned}
u_{0}(r)-u_{2}(r) & =-2 \ln \left(1+\frac{\lambda \mu_{\lambda}^{2}}{r^{2}}\right)+O\left(\left(\lambda \mu_{\lambda}^{2}\right)^{\alpha}\right) \\
& =O\left(\left(\lambda \mu_{\lambda}^{2}\right)^{\alpha}\right) .
\end{aligned}
$$

Arguing in a similar way, one shows that

$$
u_{0}^{\prime}(r)-u_{2}^{\prime}(r)=O\left(\frac{\lambda \mu_{\lambda}^{2}}{\delta^{3}}\right)+O\left(\left(\lambda \mu_{\lambda}^{2}\right)^{\alpha}\right)=O\left(\left(\lambda \mu_{\lambda}^{2}\right)^{\alpha}\right) .
$$

We will now look for a solution of (1.1) of the form $U+\phi$, where $\phi$ is a correction term. Let us observe that $U+\phi$ is a solution to (1.1) if and only if $\phi$ solves

$$
\left\{\begin{array}{l}
L(\phi)=N(\phi)-R(U) \text { in }(0,1) \\
\phi^{\prime}(0)=0 \\
\phi^{\prime}(1)=0
\end{array}\right.
$$

where

$$
\begin{aligned}
L(\phi) & =-\Delta \phi+\phi-\lambda e^{U} \phi \\
N(\phi) & =\lambda\left(e^{U+\phi}-e^{U}-e^{U} \phi\right) \\
R(U) & =-\Delta U+U-\lambda e^{U}
\end{aligned}
$$

\section{THE ERROR ESTIMATE}

In this section we estimate the terms $R(U)$ and $N(\phi)$. In order to take benefit of the estimates in [PV15], we are going to work with the norm $\|\cdot\|_{*}$ (see (5.1)) which is,roughly speaking, a weighted $L^{\infty}$-norm on $B_{\frac{1}{2}}$ and a $L^{1}$-norm elsewhere. We begin by estimating $N(\phi)$.

Lemma 4.1. There exists $C>0$ such that, for any $\beta>0$,

$$
|N(\phi)| \leq C|\phi|^{2}\left\{\begin{array}{cl}
\frac{1}{\lambda \mu_{\lambda}^{2}\left(1+\left(\frac{r}{\sqrt{\lambda} \mu_{\lambda}}\right)^{2}\right)^{2}} & \text { if } r \leq \delta \\
\varepsilon_{\lambda}^{\beta} & \text { if } \delta \leq r \leq 1-2 \delta_{1}
\end{array}\right.
$$

and

$$
\|N(\phi)\|_{L^{1}\left(B_{1} \backslash B_{\frac{1}{2}}\right)} \leq C \varepsilon_{\lambda}^{-1}\|\phi\|_{L^{\infty}\left(B_{1} \backslash B_{\frac{1}{2}}\right)}^{2} .
$$


Proof. First, using a Taylor's expansion, it is immediate to see that

$$
|N(\phi)| \leq C \lambda e^{U}|\phi|^{2} .
$$

Therefore, the proof reduces to estimate $e^{U}$. Let us observe that if $r \in[0,2 \delta]$, using (3.19) and (3.17), we have that

$$
\lambda e^{u_{0}}=\lambda e^{V_{\mu_{\lambda}}+H_{0, \mu_{\lambda}}} \leq C \frac{\mu_{\lambda}^{2}}{\left(\lambda \mu_{\lambda}^{2}+r^{2}\right)^{2}} \exp \left(\frac{r^{2}}{\varepsilon_{\lambda}}\right) \leq \frac{C}{\lambda \mu_{\lambda}^{2}\left(1+\left(\frac{r}{\sqrt{\lambda} \mu_{\lambda}}\right)^{2}\right)^{2}} .
$$

Besides, by definition of $u_{2}$, we know that it is decreasing in $(0, \tilde{r})$ and increasing elsewhere. Then, for $r \in\left[\delta, 1-2 \delta_{1}\right]$, we have

$$
e^{u_{2}(r)} \leq e^{u_{2}(\delta)}+e^{u_{2}\left(1-2 \delta_{1}\right)} .
$$

Making a Taylor's expansion, we obtain, for some $\theta \in\left(1-2 \delta_{1}, 1\right)$,

$$
u_{2}\left(1-2 \delta_{1}\right)=u_{2}(1)-2 \delta_{1} u_{2}^{\prime}(1)+2 \delta_{1}^{2} u_{2}^{\prime \prime}(\theta) \leq \frac{\sqrt{2}}{\varepsilon_{\lambda} G_{\varepsilon_{\lambda}}^{\prime}(1)}-\delta_{1} u_{2}^{\prime}(1) .
$$

Thus, noting that $u_{2}^{\prime}(1) \approx \sqrt{2} \varepsilon_{\lambda}^{-1}$ and recalling (3.3) and (3.4), we deduce that

$$
\lambda e^{u_{2}\left(1-2 \delta_{1}\right)} \leq C \varepsilon_{\lambda}^{-2} e^{-\delta_{1} u_{2}^{\prime}(1)} \leq C \varepsilon_{\lambda}^{\beta}
$$

for any $\beta>0$. On the other hand, recalling (3.8), we see that $e^{u_{2}(\delta)} \leq \frac{C}{\delta^{4}} \leq C \varepsilon_{\lambda}^{-8}$. By noticing that $\lambda \varepsilon_{\lambda}^{-8} \leq C \varepsilon_{\lambda}^{\beta}$ for any $\beta>0$, we conclude that

$$
\lambda e^{u_{2}(r)} \leq C \varepsilon_{\lambda}^{\beta}
$$

for any $r \in\left[\delta, 1-2 \delta_{1}\right]$ and any $\beta>0$. Finally, by observing that for $r \in(\delta, 2 \delta)$,

$$
\lambda e^{U} \leq \lambda \max \left\{e^{u_{0}}, e^{u_{2}}\right\} \leq C \varepsilon_{\lambda}^{\beta},
$$

we deduce (4.1).

We refer to [PV15, Lemma 4.3] for the proof of (4.2).

Next, we estimate $R(U)$.

Lemma 4.2. Let $\alpha \in\left(0, \frac{1}{2}\right)$. There exists $C>0$ such that

$$
|R(U)| \leq C \begin{cases}\frac{\left(\lambda \mu_{\lambda}^{2}\right)^{\alpha}+\frac{r^{2}}{\varepsilon_{\lambda}}+r^{2}|\ln r|}{\lambda \mu_{\lambda}^{2}\left(1+\left(\frac{r}{\sqrt{\lambda} \mu_{\lambda}}\right)^{2}\right)^{2}} & \text { if } r \leq \delta \\ \varepsilon_{\lambda}^{\beta} & \text { if } \delta \leq r \leq 1-2 \delta_{1}\end{cases}
$$


for any $\beta>0$, and

$$
\|R(U)\|_{L^{1}\left(B_{1} \backslash B_{\frac{1}{2}}\right)} \leq C \varepsilon_{\lambda}^{1+\sigma}
$$

for some $\sigma>0$.

Proof. First, we consider the case $r \leq \delta$ so that $U(r)=u_{0}(r)(r)=V_{\mu_{\lambda}}(r)+H_{0, \mu_{\lambda}}(r)$. Combining (3.1), (3.16), and (3.19), and making a Taylor's expansion, we infer that

$$
\begin{aligned}
\left|R\left(u_{0}\right)\right|= & \left|-\Delta\left(V_{\mu_{\lambda}}+H_{0, \mu_{\lambda}}\right)+V_{\mu_{\lambda}}+H_{0, \mu_{\lambda}}-\lambda e^{V_{\mu_{\lambda}}+H_{0, \mu_{\lambda}}}\right| \\
= & \left|\lambda e^{V_{\mu_{\lambda}}}\left(1-e^{H_{0, \mu_{\lambda}}}\right)\right| \\
\leq & C \lambda e^{V_{\mu_{\lambda}}}\left|H_{0, \mu_{\lambda}}\right| \\
\leq & C \frac{\left(\lambda \mu_{\lambda}^{2}\right)^{\alpha}+\frac{r^{2}}{\varepsilon_{\lambda}}+r^{2}|\ln r|}{\lambda \mu_{\lambda}^{2}\left(1+\left(\frac{r}{\sqrt{\lambda} \mu_{\lambda}}\right)^{2}\right)^{2}} .
\end{aligned}
$$

Next, when $2 \delta \leq r \leq 1-2 \delta_{1}$, we have $U(r)=u_{2}(r)$. Arguing as in the previous lemma, we obtain

$$
\left|R\left(u_{2}(r)\right)\right|=\lambda e^{u_{2}(r)} \leq C \varepsilon_{\lambda}^{\beta}
$$

for any $\beta>0$.

On the other hand, it is proven in [PV15, Lemma 4.2] that

$$
\left\|R\left(u_{4}\right)\right\|_{L^{1}\left(B_{1} \backslash B_{1-\delta_{1}}\right)} \leq C \varepsilon_{\lambda}^{1+\sigma} \text { for some } \sigma>0 .
$$

Finally, we consider the two intermediate regimes. First, let us consider the case $\delta \leq r \leq 2 \delta$. In this interval, we have $U(r)=u_{1}(r)$. Using (3.2), we get

$$
\begin{aligned}
\mid R\left(u_{1} \mid\right)= & \mid \chi_{1} R\left(u_{0}\right)+\left(1-\chi_{1}\right) R\left(u_{2}\right)-2 \chi_{1}^{\prime}\left(u_{0}^{\prime}-u_{2}^{\prime}\right)+\left(-\Delta \chi_{1}+\chi_{1}\right)\left(u_{0}-u_{2}\right) \\
& +\lambda \chi_{1} e^{u_{0}}+\lambda\left(1-\chi_{1}\right) e^{u_{2}}-\lambda e^{\chi_{1} u_{0}+\left(1-\chi_{1}\right) u_{2}} \mid \\
\leq & \left|R\left(u_{0}\right)\right|+\left|R\left(u_{2}\right)\right|+C\left(\frac{\left|u_{0}^{\prime}-u_{2}^{\prime}\right|}{\delta}+\frac{\left|u_{0}-u_{2}\right|}{\delta^{2}}\right) \\
& +\lambda e^{u_{2}}+\left|\lambda e^{u_{0}}\left(e^{\left(\chi_{1}-1\right)\left(u_{0}-u_{2}\right)}-1\right)\right| .
\end{aligned}
$$

Using a Taylor's expansion and Lemma 3.3, we have

$$
\left|\lambda e^{u_{0}}\left(e^{\left(\chi_{1}-1\right)\left(u_{0}-u_{2}\right)}-1\right)\right| \leq \lambda e^{u_{0}}\left|u_{0}-u_{2}\right| \leq \lambda e^{u_{0}}\left(\lambda \mu_{\lambda}^{2}\right)^{\alpha} .
$$

Using Lemma 3.3 once again, we get

$$
\frac{\left|u_{0}^{\prime}-u_{2}^{\prime}\right|}{\delta}+\frac{\left|u_{0}-u_{2}\right|}{\delta^{2}} \leq C\left(\lambda \mu_{\lambda}^{2}\right)^{\alpha} \delta^{-2} .
$$


Plugging these two last estimates into (4.6) and using (4.3) and (4.4), we obtain

$$
\begin{aligned}
&\left|R\left(u_{1}\right)\right| \leq C\left(\sup _{\delta \leq r \leq 2 \delta} \frac{1}{\lambda \mu_{\lambda}^{2}\left(1+\left(\frac{r}{\sqrt{\lambda_{\lambda}} \mu_{\lambda}}\right)^{2}\right)^{2}}+\varepsilon_{\lambda}^{\beta}+\frac{\left(\lambda \mu_{\lambda}^{2}\right)^{\alpha}}{\delta^{2}}\right) \\
& \leq C\left(\frac{\lambda \mu_{\lambda}^{2}}{\delta^{4}}+\varepsilon_{\lambda}^{\beta}+\frac{\left(\lambda \mu_{\lambda}^{2}\right)^{\alpha}}{\delta^{2}}\right) \leq C \varepsilon_{\lambda}^{\beta}
\end{aligned}
$$

Finally, when $1-2 \delta_{1} \leq r \leq 1-\delta_{1}$, arguing as above, we have

$$
\begin{aligned}
\left|R\left(u_{3}\right)\right|= & \mid \chi_{3} R\left(u_{2}\right)+\left(1-\chi_{3}\right) R\left(u_{4}\right)-2 \chi_{3}^{\prime}\left(u_{4}^{\prime}-u_{2}^{\prime}\right)+\left(-\Delta \chi_{3}+\chi_{3}\right)\left(u_{4}-u_{2}\right) \\
& +\lambda \chi_{3} e^{u_{4}}+\lambda\left(1-\chi_{3}\right) e^{u_{2}}-\lambda e^{\chi_{3} u_{4}+\left(1-\chi_{3}\right) u_{2} \mid} \\
\leq & \left|R\left(u_{2}\right)\right|+\left|R\left(u_{4}\right)\right|+C\left(\frac{\left|u_{4}^{\prime}-u_{2}^{\prime}\right|}{\delta_{1}}+\frac{\left|u_{4}-u_{2}\right|}{\delta_{1}^{2}}\right) \\
& +\lambda e^{u_{2}}+\lambda e^{u_{4}}\left|u_{4}-u_{2}\right| .
\end{aligned}
$$

Using Lemma 3.1 and the definition of $\delta_{1}$ given in (3.4), we obtain

$$
\int_{1-2 \delta_{1}}^{1-\delta_{1}}\left(\frac{\left|u_{4}^{\prime}-u_{2}^{\prime}\right|}{\delta_{1}}+\frac{\left|u_{4}-u_{2}\right|}{\delta_{1}^{2}}\right) r d r=O\left(\varepsilon_{\lambda}^{1+\sigma}\right)
$$

and

$$
\int_{1-2 \delta_{1}}^{1-\delta_{1}} \lambda e^{u_{4}}\left|u_{4}-u_{2}\right| r d r=O\left(\varepsilon_{\lambda}^{1+\sigma}\right) .
$$

Thanks to (4.4) and (4.5), we see that

$$
\int_{1-2 \delta_{1}}^{1-\delta_{1}}\left(R\left(u_{2}\right)+R\left(u_{4}\right)+\lambda e^{u_{2}}\right) r d r=O\left(\varepsilon_{\lambda}^{1+\sigma}\right) .
$$

Plugging the three previous estimates into (4.7), we obtain

$$
\left\|R\left(u_{3}\right)\right\|_{L^{1}\left(B_{1} \backslash B_{\frac{1}{2}}\right)} \leq C \varepsilon_{\lambda}^{1+\sigma} .
$$

This concludes the proof of the lemma.

\section{INVERTIBILITY OF THE LINEARIZED OPERATOR}

In this section we develop an invertibility theory for the operator $L$ defined in (3.21). To do so, we use ideas from [dPKM05, dPW06, dPR15, PV15]. First, we define the norms

$$
\|u\|_{*}=\max \left\{\left|\ln \left(\lambda \mu_{\lambda}^{2}\right)\right|\left\|\tilde{\chi}_{1} u\right\|_{\star},\left\|\tilde{\chi}_{2} u\right\|_{L^{1}\left(B_{1} \backslash B_{1 / 4}\right)}\right\}
$$


and

$$
\|u\|_{* *}=\max \left\{\left\|\tilde{\chi}_{1} u\right\|_{\star},\left\|\tilde{\chi}_{2} u\right\|_{L^{1}\left(B_{1} \backslash B_{1 / 4}\right)}\right\}
$$

where

$$
\tilde{\chi}_{1}(r)=\left\{\begin{array}{l}
1 \text { if } r \leq \frac{1}{2} \\
0 \text { if } r \geq \frac{3}{4}
\end{array}, \quad \tilde{\chi}_{2}(r)=\left\{\begin{array}{ll}
1 & \text { if } r \geq \frac{1}{2} \\
0 & \text { if } r \leq \frac{1}{4}
\end{array},\right.\right.
$$

and

$$
\|u\|_{\star}=\sup _{r \in\left[0, \frac{3}{4}\right]} \frac{\lambda \mu_{\lambda}^{2}|u(r)|}{\lambda \mu_{\lambda}^{2}+\left(1+\frac{r}{\sqrt{\lambda} \mu_{\lambda}}\right)^{-2-\nu}}=\sup f_{\lambda}(r)|u(r)|
$$

for some $\nu \in(0,1)$.

The following proposition is the main result of this section.

Proposition 5.1. There exist positive constants $\lambda_{0}$ and $C$ such that for any $\lambda \in$ $\left(0, \lambda_{0}\right)$ and for any $h \in L^{\infty}\left(B_{1}\right)$, there exists a unique radial function $\phi \in W^{2,2}\left(B_{1}\right)$ solution to

$$
\left\{\begin{array}{l}
L(\phi)=h \text { in } B_{1} \\
\phi^{\prime}(1)=0
\end{array}\right.
$$

which satisfies

$$
\|\phi\|_{L^{\infty}\left(B_{1}\right)} \leq C\|h\|_{*} .
$$

Rather than directly proving this statement, we first provide a priori estimates for the solution to (5.2) when $\phi$ is orthogonal to

$$
Z_{0}(r)=\frac{r^{2}-\lambda \mu_{\lambda}^{2}}{r^{2}+\lambda \mu_{\lambda}^{2}}
$$

This function is a solution to

$$
-\Delta Z_{0}=\frac{8 \lambda \mu_{\lambda}^{2}}{\left(\lambda \mu_{\lambda}^{2}+r^{2}\right)^{2}} Z_{0}
$$

that is, the linearization of the equation $-\Delta v=e^{v}$ around the radial solution

$$
v(r)=V_{\mu_{\lambda}}(r)+\ln \lambda=\ln \frac{8 \lambda \mu_{\lambda}^{2}}{\left(\lambda \mu_{\lambda}^{2}+|r|^{2}\right)^{2}} .
$$

It is well-known that the only bounded radial solutions to (5.4) are multiples of $z_{0}$ (see [CL02, Lemma 2.1]).

Consider a large but fixed number $R_{0}>0$ and a radial smooth cut-off function $\chi_{\lambda}(r)$ such that $\chi_{\lambda}(r)=1$ if $r \leq R_{0} \sqrt{\lambda} \mu_{\lambda}$ and $\chi_{\lambda}(r)=0$ if $r>\left(R_{0}+1\right) \sqrt{\lambda} \mu_{\lambda}$. 
Lemma 5.1. There exist positive constants $\lambda_{0}$ and $C$ such that for, any $\lambda \in\left(0, \lambda_{0}\right)$, the unique radial solution $\phi \in W^{2,2}\left(B_{1}\right)$ to

$$
\left\{\begin{aligned}
L(\phi) & =h \text { in } B_{1} \\
\phi^{\prime}(1) & =0 \\
\int_{B_{1}} \chi_{\lambda} Z_{0} \phi d x & =0
\end{aligned}\right.
$$

satisfies

$$
\|\phi\|_{L^{\infty}\left(B_{1}\right)} \leq C\|h\|_{* *} .
$$

Proof. Assume towards a contradiction that there exist a sequence of positive numbers $\lambda_{n} \rightarrow 0$ and a sequence of solutions $\phi_{n}$ to (5.5) such that

$$
\left\|\phi_{n}\right\|_{L^{\infty}\left(B_{1}\right)}=1 \text { and }\left\|h_{n}\right\|_{* *} \underset{n \rightarrow \infty}{\longrightarrow} 0 .
$$

We denote by $\varepsilon_{n}$ (resp. $\mu_{n}$ ) the sequence defined by (3.3) (resp. (3.10)) with $\lambda=\lambda_{n}$. Also, we let $U_{n}$ denote the first approximation for $\lambda=\lambda_{n}$ and $u_{0}^{n}$ (resp. $u_{2}^{n}$ ) the sequence defined by (3.15) (resp. (3.8)) with $\lambda=\lambda_{n}$.

Our goal is to prove that $\phi_{n}(r)=o_{n}(1)$, for any $r \in[0,1]$, which yields to a contradiction with (5.6), where here and in the rest of the proof $o_{n}(1)$ denotes a function $f_{n}(r)$ such that $\lim _{n \rightarrow \infty} f_{n}(r)=0$ uniformly in $r$. We split the proof in 4 steps.

Step 1. There holds $\phi_{n}(r)=o_{n}(1)$ on compact subsets of $(0,1)$.

First, it is easy to see that $\lambda_{n} e^{U_{n}}=o_{n}(1)$ on compact subsets of $(0,1)$. Since, by assumption, $\left\|h_{n}\right\|_{* *} \rightarrow 0$, we deduce that, up to subsequence, $\phi_{n} \rightarrow \hat{\phi} C_{2}$-uniformly on compact subsets of $(0,1)$, where $\hat{\phi}$ is a radial bounded solution to

$$
\left\{\begin{aligned}
-\Delta \hat{\phi}+\hat{\phi} & =0 \\
\hat{\phi}^{\prime}(1) & =0 .
\end{aligned} \text { in } B_{1} \backslash\{0\}\right.
$$

We claim that $\hat{\phi} \equiv 0$, which in turn implies that $\phi_{n}(r)=o_{n}(1)$ on compact subsets of $(0,1)$. To prove the claim, let us observe that equation (5.7) corresponds to the modified zero-order Bessel differential equation, whose solution is given by

$$
\hat{\phi}(r)=C_{1} \xi(r)+C_{2} \zeta(r)
$$

where $C_{1}, C_{2}$ are constants, $\xi(r)$ is the zero-order modified Bessel function of the first kind, and $\zeta(r)$ is the zero-order modified Bessel function of the second kind defined in Lemma 2.1. Since $\zeta(r)$ becomes unbounded as $r \rightarrow 0$ and $\hat{\phi}$ is bounded, we deduce that $C_{2}=0$. Moreover, since $\xi^{\prime}(1) \neq 0=\hat{\phi}^{\prime}(1)$, we have $C_{1}=0$. Hence, $\hat{\phi}=0$.

Step 2. We have that $\phi_{n}(r)=o_{n}(1)$ for $r$ close to 1 . 
We set $\psi_{n}(s)=\phi_{n}\left(\varepsilon_{n} s+1\right)$ for $s \in\left[-\varepsilon_{n}^{-1}, 0\right]$. Then, since $\psi_{n}$ is bounded, by arguing as in [PV15, Proposition 5.1] it is possible to show that $\psi_{n} \rightarrow \psi C^{2}$ uniformly on compact subsets of $(-\infty, 0]$ where $\psi$ satisfies

$$
\left\{\begin{aligned}
-\psi^{\prime \prime} & =e^{\psi} \text { in } \mathbb{R}^{-} \\
\psi^{\prime}(0) & =0 \\
\|\psi\|_{L^{\infty}} & \leq 1
\end{aligned}\right.
$$

We know (see [Gro06]) that any solution $\psi$ to $-\psi^{\prime \prime}=e^{\psi}$ is of the form

$$
\psi(s)=a \frac{e^{\sqrt{2} s}-1}{e^{\sqrt{2} s}+1}+b\left(-2+\sqrt{2} s \frac{e^{\sqrt{2} s}-1}{e^{\sqrt{2} s}+1}\right)
$$

for some $a, b \in \mathbb{R}$. Since $\|\psi\|_{\infty} \leq 1$, we have $b=0$, and since $\psi^{\prime}(0)=0$, we get $a=0$. Hence, $\psi \equiv 0$.

Let us now consider the radial Green's function $G(r, t)$ associated to the operator

$$
(-\Delta \cdot+\cdot)
$$

satisfying $G\left(r, \frac{1}{2}\right)=G^{\prime}(r, 1)=0$ and singular at the point $r \in\left(\frac{1}{2}, 1\right)$. Using Green's formula, we have that, for $\frac{1}{2}<r<1$,

$$
\begin{aligned}
\phi_{n}(r)-G^{\prime}\left(r, \frac{1}{2}\right) \phi_{n}\left(\frac{1}{2}\right) & =\int_{\frac{1}{2}}^{1} G(r, t) h_{n}(t) d t+\lambda_{n} \int_{\frac{1}{2}}^{1} G(r, t) e^{U_{n}} \phi_{n}(t) d t \\
& =\int_{\frac{1}{2}}^{1} G(r, t) h_{n}(t) d t+G(r, 1) \varepsilon_{n} \lambda_{n} \int_{-\frac{1}{2 \varepsilon_{n}}}^{0} e^{U_{n}\left(\varepsilon_{n} s+1\right)} \psi_{n}(s) d s \\
& +\varepsilon_{n} \lambda_{n} \int_{-\frac{1}{2 \varepsilon_{n}}}^{0}\left(G\left(r, \varepsilon_{n} s+1\right)-G(r, 1)\right) e^{U_{n}\left(\varepsilon_{n} s+1\right)} \psi_{n}(s) d s .
\end{aligned}
$$

From Step 1 we know that $\phi_{n}\left(\frac{1}{2}\right)=o_{n}(1)$. Combining this with the fact that $G$ is bounded and $\left\|h_{n}\right\|_{* *} \rightarrow 0$ as $n \rightarrow \infty$, we have

$$
G^{\prime}\left(r, \frac{1}{2}\right) \phi_{n}\left(\frac{1}{2}\right)+\int_{\frac{1}{2}}^{1} G(r, t) h_{n}(t) d t=o_{n}(1) .
$$

Arguing as in [PV15, Proposition 5.1], one shows that

$$
\varepsilon_{n} \lambda_{n} \int_{-\frac{1}{2 \varepsilon_{n}}}^{0}\left(G\left(r, \varepsilon_{n} s+1\right)-G(r, 1)\right) e^{U_{n}\left(\varepsilon_{n} s+1\right)} \psi_{n}(s) d s=o_{n}(1) .
$$

Hence,

$$
\phi_{n}(r)=C_{n} G(r, 1)+o_{n}(1),
$$

where $C_{n}=\varepsilon_{n} \lambda_{n} \int_{-\frac{1}{2 \varepsilon_{n}}}^{0} e^{U_{n}\left(\varepsilon_{n} s+1\right)} \psi_{n}(s) d s$.

Since $\phi_{n}(1)=\psi_{n}(1)=o_{n}(1)$ and $\lim _{r \rightarrow 1} G(r, 1) \neq 0$, we deduce that $C_{n}=0$. Hence, $\phi_{n}=o_{n}(1)$ for $r \in\left[\frac{1}{2}, 1\right]$. 
In the following steps it is convenient to work with rescaled variables. We set

$$
s=\frac{r}{\sqrt{\lambda}_{n} \mu_{n}} \quad \text { for } r \in[0,1]
$$

and define

$$
\begin{aligned}
\tilde{\phi}_{n}(s) & =\phi_{n}\left(\sqrt{\lambda_{n}} \mu_{n} s\right) \\
\tilde{U}_{n}(s) & =U_{n}\left(\sqrt{\lambda_{n}} \mu_{n} s\right)+2 \ln \left(\lambda_{n} \mu_{n}\right), \\
\tilde{h}_{n}(s) & =\lambda_{n} \mu_{n}^{2} h_{n}\left(\sqrt{\lambda_{n}} \mu_{n} s\right) .
\end{aligned}
$$

Letting $\tilde{L}(\cdot)=-\Delta \cdot+\lambda_{n} \mu_{n}^{2} \cdot-e^{\tilde{U}_{n}} \cdot$, it easy to see that $\tilde{\phi}_{n}$ satisfies

$$
\tilde{L}\left(\tilde{\phi}_{n}(s)\right)=\tilde{h}_{n}(s) \text {. }
$$

We also define (with some abuse of notation)

$$
\|\tilde{h}\|_{\star}:=\sup _{s \in\left[0, \frac{1}{2 \sqrt{\lambda_{n} \mu_{n}}}\right]} \frac{\tilde{h}(s)}{\lambda_{n} \mu_{n}^{2}+(1+s)^{-2-\nu}}=\left\|h 1_{\left\{r \leq \frac{1}{2}\right\}}\right\|_{\star},
$$

for functions $\tilde{h}$ defined in the rescaled variable.

Step 3. Up to subsequence, we have that $\tilde{\phi}_{n} \rightarrow 0$ as $n \rightarrow \infty$ uniformly over compact sets of $\mathbb{R}^{2}$.

Recalling (3.19), elliptic estimates applied to (5.8) imply that, up to subsequence, $\tilde{\phi}_{n}$ converges uniformly over compact sets of $\mathbb{R}^{2}$ to a bounded solution $\tilde{\phi}$ to

$$
-\Delta \tilde{\phi}=\frac{8}{\left(1+s^{2}\right)^{2}} \tilde{\phi} \quad \text { in } \mathbb{R}^{2} .
$$

By [CL02, Lemma 2.1], we deduce that there exists a constant $C_{0}$ such that $\tilde{\phi}=$ $C_{0} \tilde{Z}_{0}(s)$, where

$$
\tilde{Z}_{0}(s)=\frac{s^{2}-1}{s^{2}+1}=Z_{0, n}\left(\sqrt{\lambda_{n}} \mu_{n} s\right) \quad \text { with } Z_{0, n}(r)=\frac{r^{2}-\lambda_{n} \mu_{n}^{2}}{r^{2}+\lambda_{n} \mu_{n}^{2}} .
$$

Let $\tilde{\chi}(s)=\chi_{\lambda_{n}}\left(\sqrt{\lambda_{n}} \mu_{n} s\right)$ where $\chi_{\lambda}$ is defined just before the statement of the lemma. Notice that it does not depend on $n$. The orthogonality condition satisfied by $\phi_{n}$ yields

$$
0=\int_{B_{1}} \chi_{\lambda_{n}} Z_{0, n} \phi_{n} d x=\lambda_{n} \mu_{n}^{2} \int_{B_{1 /\left(\sqrt{\lambda_{n}} \mu_{n}\right)}} \tilde{\chi} \tilde{Z}_{0} \tilde{\phi}_{n} d x .
$$

Passing to the limit $n \rightarrow \infty$, we find

$$
\int_{\mathbb{R}^{2}} \tilde{\chi} \tilde{Z}_{0} \tilde{\phi} d x=0
$$

which implies $C_{0}=0$. The result thus follows.

Step 4. We have that $\phi_{n}(r)=o_{n}(1)$ for $r$ close to 0 . 
This is based on a maximum principle argument. Let us show that there exists a constant $C>0$, independent of $n$, such that

$$
\left\|\tilde{\phi}_{n}\right\|_{L^{\infty}\left(B_{\tau /\left(\sqrt{\lambda_{n}} \mu_{n}\right)}\right)} \leq C\left[\sup _{s \leq R}\left|\tilde{\phi}_{n}(s)\right|+\left\|\tilde{h}_{n}\right\|_{\star}+\left|\phi_{n}(\tau)\right|\right],
$$

where $R>0$ is a large but fixed number and $\tau$ is a small but fixed number.

To prove this, we need the following version of the maximum principle. We claim that there exists a fixed number $R_{1}>0$ such that for all $R>R_{1}$,

if $\tilde{L}(\varphi)>0$ in $A_{n}:=B_{\tau /\left(\sqrt{\lambda_{n}} \mu_{n}\right)} \backslash B_{R}$ and $\varphi \geq 0$ on $\partial A_{n}$, then $\varphi \geq 0$ in $A_{n}$.

To prove this, we consider the function $\varphi_{0}(s)=1-\frac{1}{s^{\nu}}$. Observe that

$$
-\Delta \varphi_{0}=\nu(\nu+2) \frac{1}{s^{2+\nu}}
$$

Letting $\tilde{u}_{0}^{n}(s)=u_{0}^{n}\left(\sqrt{\lambda_{n}} \mu_{n} s\right)+2 \ln \left(\lambda_{n} \mu_{n}\right)$ and $\delta_{n}$ be defined by (3.17) with $\lambda=\lambda_{n}$, and using (3.19), we deduce that, for any $s \in\left[0,2 \delta_{n} / \sqrt{\lambda_{n}} \mu_{n}\right]$,

$$
e^{\tilde{u}_{0}^{n}(s)}=\frac{8}{\left(1+s^{2}\right)^{2}} e^{H_{0, \mu_{\lambda_{n}}}\left(\sqrt{\lambda_{n}} \mu_{n} s\right)} \leq \frac{C}{\left(1+s^{2}\right)^{2}} .
$$

On the other hand, letting $\tilde{u}_{2}^{n}(s)=u_{2}^{n}\left(\sqrt{\lambda_{n}} \mu_{n} s\right)+2 \ln \left(\lambda_{n} \mu_{n}\right)$, and $\tilde{r}_{n}$ be defined by (3.14) with $\lambda=\lambda_{n}$, and recalling that $u_{2}^{n}$ is increasing for $r>\tilde{r}_{n}$ and (3.10), we have, for any $s \in\left[\tilde{r}_{n} /\left(\sqrt{\lambda_{n}} \mu_{n}\right), \tau /\left(\sqrt{\lambda_{n}} \mu_{n}\right)\right]$,

$$
e^{\tilde{u}_{2}^{n}(s)} \leq e^{\tilde{u}_{2}^{n}\left(\tau /\left(\sqrt{\lambda_{n}} \mu_{n}\right)\right)}=8\left(\frac{\sqrt{\lambda_{n}} \mu_{n}}{\tau}\right)^{4} \exp \left(H_{\varepsilon_{\lambda_{n}}}(\tau)-H_{\varepsilon_{\lambda_{n}}}(0)\right) .
$$

Choosing $\tau$ small but fixed so that (3.12) holds, we have that

$$
\exp \left(H_{\varepsilon_{\lambda_{n}}}(\tau)-H_{\varepsilon_{\lambda_{n}}}(0)\right) \leq C \exp \left(\frac{\tau^{2}}{\varepsilon_{\lambda}}\right)
$$

In view of (3.13), choosing $\tau$ smaller if necessary, we have that

$$
\frac{\sqrt{\lambda_{n}} \mu_{n}}{\tau} \exp \left(H_{\varepsilon_{\lambda_{n}}}(\tau)-H_{\varepsilon_{\lambda_{n}}}(0)\right) \leq C \frac{\sqrt{\lambda_{n}} \mu_{n}}{\tau} \exp \left(\frac{\tau^{2}}{\varepsilon_{\lambda}}\right) \leq C .
$$

Plugging this into (5.12), we deduce that

$$
e^{\tilde{u}_{2}^{n}(s)} \leq e^{\tilde{u}_{2}^{n}\left(\tau /\left(\sqrt{\lambda_{n}} \mu_{n}\right)\right)} \leq C\left(\frac{\sqrt{\lambda_{n}} \mu_{n}}{\tau}\right)^{3} \leq \frac{C}{s^{3}} .
$$

Besides, arguing similarly, it is easy to check that, for any $s \in\left[\delta_{n} / \sqrt{\lambda_{n}} \mu_{n}, \tilde{r}_{n} / \sqrt{\lambda_{n}} \mu_{n}\right]$,

$$
e^{\tilde{u}_{2}^{n}(s)} \leq \frac{C}{s^{4}}
$$

From this, (5.13), and (5.11), we deduce that

$$
e^{\tilde{U}_{n}(s)} \leq \frac{C}{s^{3}}
$$


Therefore, for any $R$ is sufficiently large, we have

$$
\tilde{L}\left(\varphi_{0}\right)=-\Delta \varphi_{0}+\lambda_{n} \mu_{n}^{2} \varphi_{0}-e^{\tilde{U}_{n}} \varphi_{0} \geq \frac{\nu^{2}}{2} \frac{1}{s^{2+\nu}}+\frac{1}{2} \lambda_{n} \mu_{n}^{2}>0 \quad \text { in } A_{n}
$$

and $\varphi_{0}>0$ on $\partial A_{n}$. The claim thus follows.

Let us now prove (5.10). We define

$$
\bar{\phi}_{n}=C_{1}\left[\max _{s \in(0, R)}\left|\tilde{\phi}_{n}(s)\right|+\left\|\tilde{h}_{n}\right\|_{\star}+\left|\phi_{n}(\tau)\right|\right] \varphi_{0}
$$

for a constant $C_{1}$ independent of $n$ and $R>R_{1}$. Observe that if $C_{1} \geq \frac{4}{\nu^{2}}$ then

$$
\tilde{L}\left(\bar{\phi}_{n}\right) \geq 2\left\|\tilde{h}_{n}\right\|_{\star}\left(s^{-2-\nu}+\lambda_{n} \mu_{n}^{2}\right) \geq\left|\tilde{h}_{n}\right| \frac{2\left(s^{-2-\nu}+\lambda_{n} \mu_{n}^{2}\right)}{\left((1+s)^{-2-\nu}+\lambda_{n} \mu_{n}^{2}\right)} \geq\left|\tilde{h}_{n}\right|=\left|\tilde{L}_{\left(\tilde{\phi}_{n}\right)}\right|
$$

in $A_{n}$, since $\frac{2\left(s^{-2-\nu}+\lambda_{n} \mu_{n}^{2}\right)}{\left((1+s)^{-2-\nu}+\lambda_{n} \mu_{n}^{2}\right)} \geq 1$ for $s \in[R,+\infty)$ (taking $R$ larger if necessary).

On the other hand, if $C_{1} \geq\left(1-R^{-\nu}\right)^{-1}$, we have

$$
\bar{\phi}_{n} \geq\left|\tilde{\phi}_{n}\right| \quad \text { on } \partial A_{n} \text {. }
$$

Applying the maximum principle and observing that $\left|\varphi_{0}\right| \leq 1$, we are led to (5.10).

Finally, by noting that $\left\|\tilde{h}_{n}\right\|_{\star} \leq\left\|h_{n}\right\|_{* *}=o_{n}(1)$ (by $\left.(5.6)\right), \phi_{n}(\tau)=o_{n}(1)$ (by Step 1), and $\max _{s \in(0, R)}\left|\hat{\phi}_{n}(s)\right|=o_{n}(1)$ (by Step 3), we conclude that $\left\|\phi_{n}\right\|_{L^{\infty}\left(B_{\tau}\right)}=o_{n}(1)$. From this and Step 1 and Step 2, we deduce that $\left\|\phi_{n}\right\|_{L^{\infty}\left(B_{1}\right)}=o_{n}(1)$ which contradicts the fact that $\left\|\phi_{n}\right\|_{L^{\infty}\left(B_{1}\right)}=1$. This completes the proof.

We are now ready to prove Proposition 5.1.

Proof of Proposition 5.1. We reuse the notation introduced in the proof of the previous lemma. For a scaled function $\tilde{g}(s)=\lambda \mu_{\lambda}^{2} g\left(\sqrt{\lambda} \mu_{\lambda} s\right)$ with $s=r /\left(\sqrt{\lambda} \mu_{\lambda}\right)$, we define

$$
\|\tilde{g}\|_{* *}:=\|g\|_{* *}
$$

Let $R>R_{0}+1$ be a large fixed number, $\tau>0$ as in Step 4 of the proof of Lemma 5.1, and $\hat{z}_{0}$ be the solution to

$$
\left\{\begin{aligned}
-\Delta \hat{z}_{0} & =\frac{8}{\left(1+s^{2}\right)^{2}} \hat{z}_{0} \text { in } B_{\tau /\left(\sqrt{\lambda} \mu_{\lambda}\right)} \backslash B_{R} \\
\hat{z}_{0}(R) & =\hat{Z}_{0}(R) \\
\hat{z}_{0}\left(\tau /\left(\sqrt{\lambda} \mu_{\lambda}\right)\right) & =0,
\end{aligned}\right.
$$


where $\tilde{Z}_{0}$ is the function defined in Step 3 of the proof of Lemma 5.1. A direct computation shows that

$$
\hat{z}_{0}(s)=\tilde{Z}_{0}(s)\left[1-\frac{\int_{R}^{s} \frac{d t}{t \tilde{Z}_{0}^{2}(t)}}{\int_{R}^{\tau /\left(\sqrt{\lambda} \mu_{\lambda}\right)} \frac{d t}{t \tilde{Z}_{0}^{2}(t)}}\right] .
$$

We also let $\hat{z}_{1}$ be the solution to

$$
\left\{\begin{aligned}
-\Delta \hat{z}_{1}+\lambda \mu_{\lambda}^{2} \hat{z}_{1} & =\lambda \mu_{\lambda}^{2} \hat{z}_{0} \quad \text { in } B_{\tau /\left(\sqrt{\lambda} \mu_{\lambda}\right)} \backslash B_{R} \\
\hat{z}_{1}(R) & =0 \\
\hat{z}_{1}\left(\tau /\left(\sqrt{\lambda} \mu_{\lambda}\right)\right) & =0 .
\end{aligned}\right.
$$

Elliptic estimates immediately yield that

$$
\left\|\hat{z}_{1}\right\|_{C^{2}\left(B_{\tau /\left(\sqrt{\lambda} \mu_{\lambda}\right)} \backslash B_{R}\right)} \leq C \lambda \mu_{\lambda}^{2}
$$

We consider smooth cut-off functions $\eta_{1}(s)$ and $\eta_{2}(s)$ with the following properties: $\eta_{1}(s)=1$ for $s<R, \eta_{1}(s)=0$ for $s>R+1,\left|\eta_{1}^{\prime}(s)\right| \leq 2, \eta_{2}(s)=1$ for $s<$ $\tau /\left(2 \sqrt{\lambda} \mu_{\lambda}\right), \eta_{2}(s)=0$ for $s>\tau /\left(\sqrt{\lambda} \mu_{\lambda}\right),\left|\eta_{2}^{\prime}(s)\right| \leq C \sqrt{\lambda} \mu_{\lambda}$, and $\left|\eta_{2}^{\prime \prime}(s)\right| \leq C \lambda \mu_{\lambda}^{2}$. We also define the test function

$$
\tilde{z}_{0}=\eta_{1} \tilde{Z}_{0}+\left(1-\eta_{1}\right) \eta_{2}\left(\hat{z}_{0}-\hat{z}_{1}\right) .
$$

Let $\phi$ be a solution to (5.2). As previously, we denote $\tilde{\phi}(s)=\phi\left(\sqrt{\lambda} \mu_{\lambda} s\right)$ and we let $\tilde{\chi}(s)=\chi_{\lambda}\left(\sqrt{\lambda} \mu_{\lambda} s\right)$ where $\chi_{\lambda}$ is defined before the statement of Lemma 5.1. Next, we modify $\tilde{\phi}$ so that the orthogonality condition with respect to $\tilde{z}_{0}$ is satisfied. We let

$$
\hat{\phi}=\tilde{\phi}+A \tilde{z}_{0}
$$

where the number $A$ is such that

$$
A \int_{B_{1 /\left(\sqrt{\lambda} \mu_{\lambda}\right)}} \tilde{\chi}\left|\tilde{z}_{0}\right|^{2} d x+\int_{B_{1 /\left(\sqrt{\lambda} \mu_{\lambda}\right)}} \tilde{\chi} \tilde{z}_{0} \tilde{\phi} d x=0 .
$$

Then

$$
\tilde{L}(\hat{\phi})=\tilde{h}+A \tilde{L}\left(\tilde{z}_{0}\right)
$$

and $\int_{B_{1 /\left(\sqrt{\lambda} \mu_{\lambda}\right)}} \tilde{\chi} \tilde{z}_{0} \hat{\phi} d x=0$. Recalling (5.14), Lemma (5.1) yields

$$
\|\hat{\phi}\|_{L^{\infty}\left(\left(B_{1 /\left(\sqrt{\lambda} \mu_{\lambda}\right)}\right)\right.} \leq C\left[\|\tilde{h}\|_{* *}+|A|\left\|\tilde{L}\left(\tilde{z}_{0}\right)\right\|_{* *}\right] .
$$

Observe that $\tilde{z}_{0}=0$ for $s>\tau /\left(\sqrt{\lambda} \mu_{\lambda}\right)$. Thus, recalling (5.9), we have

$$
\left\|\tilde{L}\left(\tilde{z}_{0}\right)\right\|_{* *}=\left\|\tilde{L}\left(\tilde{z}_{0}\right)\right\|_{\star} .
$$


Let us now estimate the size of $|A|$ and $\left\|\tilde{L}\left(\tilde{z}_{0}\right)\right\|_{\star}$. Testing equation (5.17) against $\tilde{z}_{0}$ and integrating by parts, we find

$$
\left\langle\hat{\phi}, \tilde{L}\left(\tilde{z}_{0}\right)\right\rangle=\left\langle\tilde{h}, \tilde{z}_{0}\right\rangle+A\left\langle\tilde{L}\left(\tilde{z}_{0}\right), \tilde{z}_{0}\right\rangle
$$

where $\langle f, g\rangle=\int_{B_{1 /\left(\sqrt{\lambda} \mu_{\lambda}\right)}} f g d x$. Combining this with (5.18),

$$
\int_{B_{1 /\left(\sqrt{\lambda} \mu_{\lambda}\right)}}\left|\hat{\phi}\left\|\tilde{L}\left(\tilde{z}_{0}\right) \mid d x \leq C\right\| \hat{\phi}\left\|_{\infty}\right\| \tilde{L}\left(\tilde{z}_{0}\right) \|_{\star}, \quad \text { and } \quad \int_{B_{1 /\left(\sqrt{\lambda} \mu_{\lambda}\right)}}\right| \tilde{h}\left\|\tilde{z_{0}} \mid d x \leq C\right\| \tilde{h} \|_{\star}
$$

we are led to

$$
A\left\langle\tilde{L}\left(\tilde{z}_{0}\right), \tilde{z}_{0}\right\rangle \leq C\|\tilde{h}\|_{\star}\left[1+\left\|\tilde{L}\left(\tilde{z}_{0}\right)\right\|_{\star}\right]+C|A|\left\|\tilde{L}\left(\tilde{z}_{0}\right)\right\|_{\star}^{2} .
$$

We next measure the size of $\left\|\tilde{L}\left(\tilde{z}_{0}\right)\right\|_{\star}$. We have

$$
\begin{aligned}
\tilde{L}\left(\tilde{z}_{0}\right)= & \eta_{1}\left(\frac{8}{\left(1+s^{2}\right)^{2}} \tilde{Z}_{0}+\mu_{\lambda}^{2} \lambda \tilde{Z}_{0}-e^{\tilde{U}} \tilde{Z}_{0}\right)+\left(1-\eta_{1}\right) \eta_{2}\left(\frac{8}{\left(1+s^{2}\right)^{2}} \tilde{z}_{0}-e^{\tilde{U}}\left(\hat{z}_{0}-\hat{z}_{1}\right)\right) \\
& +2 \nabla \eta_{1} \nabla\left(\eta_{2}\left(\hat{z}_{0}-\hat{z}_{1}\right)-\tilde{Z}_{0}\right)+\Delta \eta_{1}\left(\eta_{2}\left(\hat{z}_{0}-\hat{z}_{1}\right)-\tilde{Z}_{0}\right) \\
& -2\left(1-\eta_{1}\right) \nabla \eta_{2} \nabla\left(\hat{z}_{0}-\hat{z}_{1}\right)-\left(1-\eta_{1}\right) \Delta \eta_{2}\left(\hat{z}_{0}-\hat{z}_{1}\right),
\end{aligned}
$$

where $\tilde{U}$ denotes, as in the proof of the previous lemma, the first approximation of the solution in the rescaled variable.

In the support of $\eta_{1}$, we have

$$
\frac{8}{\left(1+s^{2}\right)^{2}} \tilde{Z}_{0}-e^{\tilde{U}} \tilde{Z}_{0}=\frac{8}{\left(1+s^{2}\right)^{2}}\left(1-e^{\tilde{H}_{0, \mu_{\lambda}}}\right) \tilde{Z}_{0}
$$

which combined with (3.19) gives

$$
\left\|\eta_{1}\left(\frac{8}{\left(1+s^{2}\right)^{2}} \tilde{Z}_{0}+\lambda \mu_{\lambda}^{2} \tilde{Z}_{0}-e^{\tilde{U}} \tilde{Z}_{0}\right)\right\|_{\star} \leq C\left(\lambda \mu_{\lambda}^{2}\right)^{\alpha} .
$$

If $R \leq s \leq \delta /\left(\sqrt{\lambda} \mu_{\lambda}\right)$ (recall (3.17)), we have that

$$
\frac{8}{\left(1+s^{2}\right)^{2}} \tilde{z}_{0}-e^{\tilde{U}}\left(\hat{z}_{0}-\hat{z}_{1}\right)=\frac{8}{\left(1+s^{2}\right)^{2}}\left(1-e^{\tilde{H}_{0, \mu_{\lambda}}}\right) \hat{z}_{0}+\frac{8}{\left(1+s^{2}\right)^{2}} e^{\tilde{H}_{0, \mu_{\lambda}}} \hat{z}_{1} .
$$

Therefore, using once again (3.19) and (5.15), we deduce that

$$
\left\|\left(\frac{8}{\left(1+s^{2}\right)^{2}} \tilde{z}_{0}-e^{\tilde{U}}\left(\hat{z}_{0}-\hat{z}_{1}\right)\right) 1_{\left\{R \leq s \leq \delta /\left(\sqrt{\lambda} \mu_{\lambda}\right)\right\}}\right\|_{\star} \leq C\left(\lambda \mu_{\lambda}^{2}\right)^{\alpha} .
$$

Arguing as in Step 4 of the proof of Lemma (5.1), we deduce that, for any $s \in$ $\left[\delta /\left(\sqrt{\lambda} \mu_{\lambda}\right), \tau /\left(\sqrt{\lambda} \mu_{\lambda}\right)\right]$

$$
e^{\tilde{U}} \leq \frac{C}{s^{3}}
$$


Hence,

$$
\left\|\left(\frac{8}{\left(1+s^{2}\right)^{2}} \tilde{z}_{0}-e^{\tilde{U}}\left(\hat{z}_{0}-\hat{z}_{1}\right)\right) 1_{\left\{\delta /\left(\sqrt{\lambda} \mu_{\lambda}\right) \leq s \leq \tau /\left(\sqrt{\lambda} \mu_{\lambda}\right)\right\}}\right\|_{\star} \leq C\left(\lambda \mu_{\lambda}^{2}\right)^{\alpha},
$$

and therefore

$$
\begin{aligned}
& \| \eta_{1}\left(\frac{8}{\left(1+s^{2}\right)^{2}} \tilde{Z}_{0}+\lambda \mu_{\lambda}^{2} \tilde{Z}_{0}-e^{\tilde{U}} \tilde{Z}_{0}\right) \\
& \quad+\left(1-\eta_{1}\right) \eta_{2}\left(\frac{8}{\left(1+s^{2}\right)^{2}} \tilde{z}_{0}-e^{\tilde{U}}\left(\hat{z}_{0}-\hat{z}_{1}\right)\right) \|_{\star} \leq C\left(\lambda \mu_{\lambda}^{2}\right)^{\alpha} .
\end{aligned}
$$

On the other hand, it is easy to see that, for $s \in(R, R+1)$,

$$
\left|\tilde{Z}_{0}-\hat{z}_{0}\right|=\left|\tilde{Z}_{0} \frac{\int_{R}^{s} \frac{d t}{t \tilde{Z}_{0}^{2}(t)}}{\int_{R}^{\tau /\left(\sqrt{\lambda} \mu_{\lambda}\right)} \frac{d t}{t \tilde{Z}_{0}^{2}(t)}}\right| \leq C\left|\ln \left(\lambda \mu_{\lambda}^{2}\right)\right|^{-1} \quad \text { and } \quad\left|\tilde{Z}_{0}^{\prime}-\hat{z}_{0}^{\prime}\right| \leq C\left|\ln \left(\lambda \mu_{\lambda}^{2}\right)\right|^{-1} \text {. }
$$

Besides, for $s \in\left(\tau /\left(2 \sqrt{\lambda} \mu_{\lambda}\right), \tau /\left(\sqrt{\lambda} \mu_{\lambda}\right)\right)$, we have

$$
\left|\hat{z}_{0}\right| \leq C\left|\ln \left(\lambda \mu_{\lambda}^{2}\right)\right|^{-1} \text { and }\left|\hat{z}_{0}^{\prime}\right| \leq C \sqrt{\lambda} \mu_{\lambda}\left|\ln \left(\lambda \mu_{\lambda}^{2}\right)\right|^{-1}
$$

We then easily deduce, using (5.15), that

$$
\begin{aligned}
& \| 2 \nabla \eta_{1} \nabla\left(\eta_{2}\left(\hat{z}_{0}-\hat{z}_{1}\right)-\tilde{Z}_{0}\right)+\Delta \eta_{1}\left(\eta_{2}\left(\hat{z}_{0}-\hat{z}_{1}\right)-\tilde{Z}_{0}\right) \\
& -2\left(1-\eta_{1}\right) \nabla \eta_{2} \nabla\left(\hat{z}_{0}-\hat{z}_{1}\right)-\left(1-\eta_{1}\right) \Delta \eta_{2}\left(\hat{z}_{0}-\hat{z}_{1}\right) \|_{\star} \leq C\left|\ln \left(\lambda \mu_{\lambda}^{2}\right)\right|^{-1} .
\end{aligned}
$$

By combining this with (5.21), we conclude that

$$
\left\|\tilde{L}\left(\tilde{z}_{0}\right)\right\|_{\star} \leq C\left|\ln \left(\lambda \mu_{\lambda}^{2}\right)\right|^{-1}
$$

Finally, we estimate $\left\langle\tilde{L}\left(\tilde{z}_{0}\right), \tilde{z}_{0}\right\rangle$. Arguing as above, it is easy to see that

$$
\left\langle\tilde{L}\left(\tilde{z}_{0}\right), \tilde{z}_{0}\right\rangle=\int_{B_{R+1} \backslash B_{R}} \tilde{L}\left(\tilde{z}_{0}\right) \tilde{z}_{0} d x+\int_{B_{\tau /\left(\sqrt{\lambda} \mu_{\lambda}\right)} \backslash B_{\tau /\left(2 \sqrt{\lambda} \mu_{\lambda}\right)}} \tilde{L}\left(\tilde{z}_{0}\right) \tilde{z}_{0} d x+O\left(\left(\lambda \mu_{\lambda}^{2}\right)^{\alpha}\right) .
$$

Using (5.20), (5.15), and (5.22), we get

$$
\begin{aligned}
& \left|\int_{B_{\tau /\left(\sqrt{\lambda} \mu_{\lambda}\right)} \backslash B_{\tau /\left(2 \sqrt{\lambda} \mu_{\lambda}\right)}} \tilde{L}\left(\tilde{z}_{0}\right) \tilde{z}_{0} d x\right| \\
& \leq C \int_{B_{\tau /\left(\sqrt{\lambda} \mu_{\lambda}\right)} \backslash B_{\tau /\left(2 \sqrt{\lambda} \mu_{\lambda}\right)}}\left(\left|\nabla \eta_{2}\right|\left|\nabla \hat{z}_{0}\right|\left|\hat{z}_{0}\right|+\left|\Delta \eta_{2}\right|\left|\hat{z}_{0}\right|^{2}\right)+O\left(\left(\lambda \mu_{\lambda}^{2}\right)^{\alpha}\right) \\
& \leq C\left|\ln \left(\lambda \mu_{\lambda}^{2}\right)\right|^{-2} .
\end{aligned}
$$


On the other hand, we have

$$
\begin{aligned}
I:= & \int_{B_{R+1} \backslash B_{R}} \tilde{L}\left(\tilde{z}_{0}\right) \tilde{z}_{0} d x= \\
& 2 \int_{B_{R+1} \backslash B_{R}} \nabla \eta_{1} \nabla\left(\hat{z}_{0}-\tilde{Z}_{0}\right) \hat{z}_{0} d x+\int_{B_{R+1} \backslash B_{R}} \Delta \eta_{1}\left(\hat{z}_{0}-\tilde{Z}_{0}\right) \hat{z}_{0} d x+O\left(\left(\lambda \mu_{\lambda}^{2}\right)^{\alpha}\right) .
\end{aligned}
$$

Thus, integrating by parts, we find

$$
I=\int_{B_{R+1} \backslash B_{R}} \nabla \eta_{1} \nabla\left(\hat{z}_{0}-\tilde{Z}_{0}\right) \hat{z}_{0} d x-\int_{B_{R+1} \backslash B_{R}} \nabla \eta_{1}\left(\hat{z}_{0}-\tilde{Z}_{0}\right) \nabla \hat{z}_{0} d x+O\left(\left(\lambda \mu_{\lambda}^{2}\right)^{\alpha}\right) .
$$

Now, we observe that, for $s \in(R, R+1),\left|\tilde{Z}_{0}(s)-\hat{z}_{0}(s)\right| \leq C\left|\ln \left(\lambda \mu_{\lambda}^{2}\right)\right|^{-1}$, while $\left|\hat{z}_{0}^{\prime}(s)\right| \leq C\left(\frac{1}{R^{3}}+\frac{1}{R}\left|\ln \left(\lambda \mu_{\lambda}^{2}\right)\right|^{-1}\right)$. Thus

$$
\left|\int_{B_{R+1} \backslash B_{R}} \nabla \eta_{1}\left(\hat{z}_{0}-\tilde{Z}_{0}\right) \nabla \tilde{z}_{0} d x\right| \leq \frac{D}{R^{3}}\left|\ln \left(\lambda \mu_{\lambda}^{2}\right)\right|^{-1}
$$

where $D$ is a constant that does not depend on $R$. Note that

$$
\begin{aligned}
\int_{B_{R+1} \backslash B_{R}} & \nabla \eta_{1} \nabla\left(\hat{z}_{0}-\tilde{Z}_{0}\right) \hat{z}_{0} d x \\
& =2 \pi \int_{R}^{R+1} \eta_{1}^{\prime}\left(\hat{z}_{0}-\tilde{Z}_{0}\right)^{\prime}\left(\tilde{Z}_{0}+O\left(\left|\ln \left(\lambda \mu_{\lambda}^{2}\right)\right|^{-1}\right)\right) s d s \\
& =-\frac{2 \pi}{\int_{R}^{\tau /\left(\sqrt{\lambda} \mu_{\lambda}\right)} \frac{d t}{t \tilde{Z}_{0}^{2}(t)}} \int_{R}^{R+1} \eta_{1}^{\prime}(s)\left(\tilde{Z}_{0}(s) \int_{R}^{s} \frac{d z}{z \tilde{Z}_{0}^{2}(z)}+\frac{1}{s}\right) d s\left[1+O\left(\left|\ln \left(\lambda \mu_{\lambda}^{2}\right)\right|^{-1}\right)\right] \\
& =-\frac{E}{\left|\ln \left(\lambda \mu_{\lambda}^{2}\right)\right|}\left[1+o_{R}(1)+O\left(\left|\ln \left(\lambda \mu_{\lambda}^{2}\right)\right|^{-1}\right)\right]
\end{aligned}
$$

where $E$ is a strictly positive constant independent of $R$ and $\lambda$. We thus conclude, choosing $R$ large enough, that $I \sim-E\left|\ln \left(\lambda \mu_{\lambda}^{2}\right)\right|^{-1}$. Combining this with (5.24), we find

$$
\left\langle\tilde{L}\left(\tilde{z}_{0}\right), \tilde{z}_{0}\right\rangle=-\frac{E}{\left|\ln \left(\lambda \mu_{\lambda}^{2}\right)\right|}\left[1+O\left(\left|\ln \left(\lambda \mu_{\lambda}^{2}\right)\right|^{-1}\right)\right] .
$$

This together with (5.19) and (5.23), yields

$$
|A| \leq C\left|\ln \left(\lambda \mu_{\lambda}^{2}\right)\right|\|\tilde{h}\|_{\star} .
$$

Recalling (5.16) and using (5.18), we then deduce that

$$
\|\phi\|_{L^{\infty}\left(B_{1}\right)} \leq C\left(\|\tilde{h}\|_{* *}+\left|\ln \left(\lambda \mu_{\lambda}^{2}\right)\right|\|\tilde{h}\|_{\star}\right) .
$$

Observe that

$$
\|\tilde{h}\|_{\star}=\sup _{s \in\left[0,1 /\left(2 \sqrt{\lambda} \mu_{\lambda}\right)\right]} \frac{\tilde{h}(s)}{\lambda \mu_{\lambda}^{2}+(1+s)^{-2-\nu}} \leq \sup _{r \in[0,1 / 2]} \frac{\lambda \mu_{\lambda}^{2}|h(r)|}{\lambda \mu_{\lambda}^{2}+\left(1+\frac{r}{\sqrt{\lambda} \mu_{\lambda}}\right)^{-2-\nu}} \leq\left\|\tilde{\chi}_{1} h\right\|_{\star} .
$$


The previous two inequalities then yield

$$
\|\phi\|_{L^{\infty}\left(B_{1}\right)} \leq C\left(\|h\|_{* *}+\left|\ln \left(\lambda \mu_{\lambda}^{2}\right)\right|\left\|\chi_{1} h\right\|_{\star}\right) .
$$

Recalling the definition of the norm $\|\cdot\|_{*}$, we conclude that

$$
\|\phi\|_{L^{\infty}\left(B_{1}\right)} \leq C\|h\|_{*} .
$$

It only remains to prove the existence part of the statement. For this purpose, we consider the space

$$
H=\left\{\phi \in H^{1}\left(B_{1}\right) \mid \phi \text { is radial }\right\},
$$

endowed with the inner product $\langle\phi, \psi\rangle_{H^{1}}=\int_{B_{1}} \nabla \phi \nabla \psi d x+\int_{B_{1}} \phi \psi d x$. Problem (5.2) expressed in weak form is equivalent to finding $\phi \in H$ such that

$$
\langle\phi, \psi\rangle_{H^{1}}=\int_{B_{1}}\left[\lambda e^{U} \phi+h\right] \psi d x \quad \text { for all } \psi \in H .
$$

By Fredholm's alternative this is equivalent to the uniqueness of solutions to this problem, which is guaranteed by (5.3).

\section{The Proof of TheOrem 1.1}

Proof. Thanks to Proposition 5.1, we know that the operator $L$ is invertible. Therefore, we can rewrite $(3.20)$ as

$$
\phi=T(\phi)=L^{-1}[R(U)+N(\phi)] .
$$

Let $\rho$ be a fixed number. We define

$$
A_{\rho}=\left\{\phi \in L^{\infty}\left(B_{1}\right):\|\phi\|_{L^{\infty}\left(B_{1}\right)} \leq \rho \varepsilon_{\lambda}^{1+\sigma}\right\}
$$

where $\sigma$ is the constant defined in Lemma 4.2. We will show that the map $T: A_{\rho} \rightarrow$ $A_{\rho}$ is a contraction. Using Lemma 4.1, recalling the definition of $\|\cdot\|_{*}$ given in (5.1), and since $\left|\ln \left(\lambda \mu_{\lambda}^{2}\right)\right|=O\left(\varepsilon_{\lambda}^{-1}\right)$, we see that

$$
\begin{aligned}
\left\|\lambda e^{U}\right\|_{*} \leq C \max & \left|\ln \left(\lambda \mu_{\lambda}^{2}\right)\right| \sup _{r \leq \delta} f_{\lambda}(r) \frac{1}{\lambda \mu_{\lambda}^{2}\left(1+\left(\frac{r}{\sqrt{\lambda} \mu_{\lambda}}\right)^{2}\right)^{2}}, \\
& \left.\left|\ln \left(\lambda \mu_{\lambda}^{2}\right)\right| \sup _{\delta \leq r \leq 3 / 4} f_{\lambda}(r) \varepsilon_{\lambda}^{\beta}, \varepsilon_{\lambda}^{-1}\right) \\
\leq C \varepsilon_{\lambda}^{-1} . &
\end{aligned}
$$

From this and recalling the definition of $N(\cdot)$ (see $(3.22)$ ), we deduce that, for $\phi, \psi \in A_{\rho}$,

$$
\|N(\phi)\|_{*} \leq\left\|\lambda e^{U}\right\|_{*}\|\phi\|_{L^{\infty}\left(B_{1}\right)}^{2} \leq C \varepsilon_{\lambda}^{-1}\|\phi\|_{L^{\infty}\left(B_{1}\right)}^{2}
$$


and

$$
\|N(\phi)-N(\psi)\|_{*} \leq C \varepsilon_{\lambda}^{-1} \max \left\{\|\phi\|_{L^{\infty}\left(B_{1}\right)},\|\psi\|_{L^{\infty}\left(B_{1}\right)}\right\}\|\phi-\psi\|_{L^{\infty}\left(B_{1}\right)} .
$$

Next, using Lemma 4.2, we obtain

$$
\begin{aligned}
\|R(U)\|_{*} \leq C \max \left(\left|\ln \left(\lambda \mu_{\lambda}^{2}\right)\right| \sup _{r \leq \delta} f_{\lambda}(r) \frac{\frac{r^{2}}{\varepsilon_{\lambda}}+r^{2}|\ln r|+\left(\lambda \mu_{\lambda}^{2}\right)^{\alpha}}{\lambda \mu_{\lambda}^{2}\left(1+\left(\frac{r}{\sqrt{\lambda} \mu_{\lambda}}\right)^{2}\right)^{2}},\right. \\
\left.\left|\ln \left(\lambda \mu_{\lambda}^{2}\right)\right| \sup _{\delta \leq r \leq 3 / 4} f_{\lambda}(r) \varepsilon_{\lambda}^{\beta}, \varepsilon_{\lambda}^{1+\sigma}\right) \\
\leq C \varepsilon_{\lambda}^{1+\sigma} .
\end{aligned}
$$

Thus, using (6.1) and (6.2), we get that, for $\phi, \psi \in A_{\rho}$,

$$
\|T(\phi)\|_{L^{\infty}\left(B_{1}\right)} \leq C\left(\|N(\phi)\|_{*}+\|R(U)\|_{*}\right) \leq C \varepsilon_{\lambda}^{1+\sigma}\left(\rho^{2} \varepsilon_{\lambda}^{\sigma}+1\right)
$$

and

$$
\|T(\phi)-T(\psi)\|_{L^{\infty}\left(B_{1}\right)} \leq C\|N(\phi)-N(\psi)\|_{*} \leq C \rho \varepsilon_{\lambda}^{\sigma}\|\phi-\psi\|_{L^{\infty}\left(B_{1}\right)},
$$

where $C$ is a constant independent of $\rho$. It follows that, for any sufficiently small $\lambda$ (and thus $\varepsilon_{\lambda}$ ), $T$ is a contraction mapping in $A_{\rho}$, and it therefore has a unique fixed point in $A_{\rho}$ for $\rho>2 C$. This concludes the proof.

\section{Appendix A. An elliptic estimate}

We show a very rough elliptic estimate which is needed in the proof of Lemma 3.2 .

Lemma A.1. Let $R>0$ and $u \in H^{1}\left(B_{R}(0)\right)$ be a radial solution to

$$
\left\{\begin{aligned}
-\Delta u+u & =f \quad \text { in } B_{R}(0) \\
u^{\prime}(R) & =g
\end{aligned}\right.
$$

for some $f \in L^{q}\left(B_{R}(0)\right)$, with $q>2$. Then, we have

$$
\|u\|_{L^{\infty}\left(B_{R}(0)\right)} \leq C\left[\left(\frac{1}{R}+R\right) R^{1-2 / q}\|f\|_{L^{q}\left(B_{R}(0)\right)}+\left(\frac{1}{R}+R^{2}\right)\|g\|_{L^{\infty}\left(\partial B_{R}(0)\right)}\right]
$$

and

$$
\left\|u^{\prime}\right\|_{L^{\infty}\left(B_{R}(0)\right)} \leq C\left[R^{1-2 / q}\|f\|_{L^{q}\left(B_{R}(0)\right)}+(1+R)\|g\|_{L^{\infty}\left(\partial B_{R}(0)\right)}\right]
$$

for some constant $C$ not depending on $R$. 
Proof. Multiplying the equation by $u$ and integrating by parts, we get

$$
\|u\|_{H^{1}\left(B_{R}\right)}^{2} \leq\|f\|_{L^{2}\left(B_{R}\right)}\|u\|_{H^{1}\left(B_{R}\right)}+R\left|u^{\prime}(R) \| u(R)\right| .
$$

Since $u(R)-u(r)=\int_{r}^{R} u^{\prime}(s) d s$, one deduces that

$$
|u(R)|^{2} \leq C\left[|u(r)|^{2}+\left\|u^{\prime}\right\|_{L^{2}\left(B_{R}\right)}^{2} \ln \frac{R}{r}\right]
$$

where throughout the proof $C$ denotes a constant not depending on $R$. Multiplying the previous inequality by $r$ and integrating, we find

$$
R^{2}|u(R)|^{2} \leq C\left[\|u\|_{L^{2}\left(B_{R}\right)}^{2}+\left\|u^{\prime}\right\|_{L^{2}\left(B_{R}\right)}^{2} R^{2}\right] .
$$

This implies that

$$
|u(R)| \leq C\left(\frac{1}{R}+1\right)\|u\|_{H_{1}\left(B_{R}\right)} .
$$

From (A.1), (A.2), and $u^{\prime}(R)=g$, we obtain that

$$
\|u\|_{H^{1}\left(B_{R}\right)}^{2} \leq\|f\|_{L^{2}\left(B_{R}\right)}\|u\|_{H^{1}\left(B_{R}\right)}+C(1+R)\|g\|_{L^{\infty}\left(\partial B_{R}\right)}\|u\|_{H^{1}\left(B_{R}\right)} .
$$

Thanks to Hölder inequality, we find that

$$
\|u\|_{H^{1}\left(B_{R}\right)} \leq C\left[R^{1-2 / q}\|f\|_{L^{q}\left(B_{R}\right)}+(1+R)\|g\|_{L^{\infty}\left(\partial B_{R}\right)}\right] .
$$

Next, observe that for any $s \in(0, R)$ we can rewrite the equation as

$$
u^{\prime}(s) s=\int_{0}^{s}(u-f) r d r
$$

From Hölder inequality, we obtain that

$$
\left|u^{\prime}(s)\right| \leq C\|u-f\|_{L^{2}\left(B_{R}\right)} \leq C\left(\|u\|_{L^{2}\left(B_{R}\right)}+R^{1-2 / q}\|f\|_{L^{q}\left(B_{R}\right)}\right) .
$$

From (A.3), we deduce that

$$
\left\|u^{\prime}\right\|_{L^{\infty}\left(B_{R}\right)} \leq C\left(R^{1-2 / q}\|f\|_{L^{q}\left(B_{R}\right)}+(1+R)\|g\|_{L^{\infty}\left(\partial B_{R}\right)}\right) .
$$

By noting that

$$
u(R)-u(\tilde{s})=\int_{\tilde{s}}^{R} u^{\prime}(r) d r
$$

we get from (A.2) that

$$
\begin{aligned}
\|u\|_{L^{\infty}\left(B_{R}\right)} & \leq C\left[\left(\frac{1}{R}+1\right)\|u\|_{H^{1}\left(B_{R}\right)}+R\left\|u^{\prime}\right\|_{L^{\infty}\left(B_{R}\right)}\right] \\
& \leq C\left[\left(\frac{1}{R}+1+R\right) R^{1-2 / q}\|f\|_{L^{q}\left(B_{R}\right)}+\left(\frac{1}{R}+R^{2}\right)\|g\|_{L^{\infty}\left(\partial B_{R}\right)}\right] .
\end{aligned}
$$

This concludes the proof. 


\section{REFERENCES}

[AP16] O. Agudelo and A. Pistoia, Boundary concentration phenomena for the higherdimensional Keller-Segel system, Calc. Var. Partial Differential Equations 55 (2016), no. 6, Paper No. 132, 31. MR3566211 $\uparrow 4$

[AS64] M. Abramowitz and I. A. Stegun, Handbook of mathematical functions with formulas, graphs, and mathematical tables, Dover, New York, 1964. $\uparrow 7$

[BBTW15] N. Bellomo, A. Bellouquid, Y. Tao, and M. Winkler, Toward a mathematical theory of Keller-Segel models of pattern formation in biological tissues, Math. Models Methods Appl. Sci. 25 (2015), no. 9, 1663-1763. MR3351175 22

[BCN17a] D. Bonheure, J.-B. Casteras, and B. Noris, Layered solutions with unbounded mass for the Keller-Segel equation, J. Fixed Point Theory Appl. 19 (2017), no. 1, 529-558. MR3625083 $\uparrow 5,10,12$

[BCN17b] D. Bonheure, J.-B. Casteras, and B. Noris, Multiple positive solutions of the stationary Keller-Segel system, Calc. Var. Partial Differential Equations 56 (2017), no. 3, Paper No. 74,35 . MR3641921 $\uparrow 4,5,7$

[CL02] C.-C. Chen and C.-S. Lin, Sharp estimates for solutions of multi-bubbles in compact Riemann surfaces, Comm. Pure Appl. Math. 55 (2002), no. 6, 728-771. MR1885666 $\uparrow 19,22$

[dPKM05] M. del Pino, M. Kowalczyk, and M. Musso, Singular limits in Liouville-type equations, Calc. Var. Partial Differential Equations 24 (2005), no. 1, 47-81. MR2157850 18

[dPMRW19] M. del Pino, M. Musso, C. Román, and J. Wei, Interior bubbling solutions for the critical Lin-Ni-Takagi problem in dimension 3, J. Anal. Math. 137 (2019), no. 2, 813-843. MR3938021 $\uparrow 3$

[dPPV16] M. del Pino, A. Pistoia, and G. Vaira, Large mass boundary condensation patterns in the stationary Keller-Segel system, J. Differential Equations 261 (2016), no. 6, 3414-3462. MR3527634 个4

[dPR15] M. del Pino and C. Román, Large conformal metrics with prescribed sign-changing Gauss curvature, Calc. Var. Partial Differential Equations 54 (2015), no. 1, 763-789. MR3385180 18

[dPW06] M. del Pino and J. Wei, Collapsing steady states of the Keller-Segel system, Nonlinearity 19 (2006), no. 3, 661-684. MR2209293 (2007b:35130) $\uparrow 3,5,13,18$

[Gro06] M. Grossi, Asymptotic behaviour of the Kazdan-Warner solution in the annulus, J. Differential Equations 223 (2006), no. 1, 96-111. MR2210140 (2006m:35122) $\uparrow 21$

[Hor03] D. Horstmann, From 1970 until present: the Keller-Segel model in chemotaxis and its consequences. I, Jahresber. Deutsch. Math.-Verein. 105 (2003), no. 3, 103-165. MR2013508 $\uparrow 2$

[Hor04] D. Horstmann, From 1970 until present: the Keller-Segel model in chemotaxis and its consequences. II, Jahresber. Deutsch. Math.-Verein. 106 (2004), no. 2, 51-69. MR2073515 $\uparrow 2$

[KS70] E. F. Keller and L. A. Segel, Initiation of slime mold aggregation viewed as an instability, Journal of Theoretical Biology 26 (1970), no. 3, 399-415. $\uparrow 1$

[LN88] C. S. Lin and W.-M. Ni, On the diffusion coefficient of a semilinear Neumann problem, Calculus of variations and partial differential equations (Trento, 1986), 1988, pp. 160174. MR974610 $\uparrow 2$

[LNT88] C.-S. Lin, W.-M. Ni, and I. Takagi, Large amplitude stationary solutions to a chemotaxis system, J. Differential Equations 72 (1988), no. 1, 1-27. MR929196 ^2 
[Ni04] W.-M. Ni, Qualitative properties of solutions to elliptic problems, Stationary partial differential equations. Vol. I, 2004, pp. 157-233. MR2103689 ^3

[NT86] W.-M. Ni and I. Takagi, On the Neumann problem for some semilinear elliptic equations and systems of activator-inhibitor type, Trans. Amer. Math. Soc. 297 (1986), no. 1, 351-368. MR849484 $\uparrow 2$

[PV15] A. Pistoia and G. Vaira, Steady states with unbounded mass of the Keller-Segel system, Proc. Roy. Soc. Edinburgh Sect. A 145 (2015), no. 1, 203-222. MR3304582 ^4, 5, $10,15,16,17,18,21$

[Sch85] R. Schaaf, Stationary solutions of chemotaxis systems, Trans. Amer. Math. Soc. 292 (1985), no. 2, 531-556. MR808736 (87a:35020) $\uparrow 3$

[SS00] T. Senba and T. Suzuki, Some structures of the solution set for a stationary system of chemotaxis, Adv. Math. Sci. Appl. 10 (2000), no. 1, 191-224. MR1769174 (2001d:35068) $\uparrow 3$

[SS02] T. Senba and T. Suzuki, Weak solutions to a parabolic-elliptic system of chemotaxis, J. Funct. Anal. 191 (2002), no. 1, 17-51. MR1909263 (2003c:35155) $\uparrow 3$

[SW13] S. Santra and J. Wei, New entire positive solution for the nonlinear Schrödinger equation: coexistence of fronts and bumps, Amer. J. Math. 135 (2013), no. 2, 443491. MR3038717 55

[WW02] G. Wang and J. Wei, Steady state solutions of a reaction-diffusion system modeling chemotaxis, Math. Nachr. 233/234 (2002), 221-236. MR1879873 (2003a:35078) $\uparrow 3$

[WW08] L. Wang and J. Wei, Solutions with interior bubble and boundary layer for an elliptic problem, Discrete Contin. Dyn. Syst. 21 (2008), no. 1, 333-351. MR2379470 个5

[WW14] J. Wei and M. Winter, Mathematical aspects of pattern formation in biological systems, Applied Mathematical Sciences, vol. 189, Springer, London, 2014. MR3114654 $\uparrow 3$

Département de Mathématique, Université libre de Bruxelles, Campus de la Plaine CP 213, Bd. Du Triomphe, 1050 Bruxelles, Belgium

Email address: denis.bonheure@ulb.ac.be

CMafCio, Faculdade de Ciências da Universidade de Lisboa, Edificio C6, Piso 1, Campo Grande 1749-016 Lisboa, Portugal

Email address: jeanbaptiste.casteras@gmail.com

Facultad de Matemáticas, Pontificia Universidad Católica de Chile, Vicuña Mackenna 4860, 7820436 Macul, Santiago, Chile

Email address: carlos.roman@mat.uc.cl 